\title{
Dynamic nuclear polarization at high magnetic fields
}

\author{
Thorsten Maly $^{1}$, Galia T. Debelouchina ${ }^{1}$, Vikram S. Bajaj ${ }^{1}$, Kan-Nian Hu${ }^{1}$, Chan-Gyu Joo ${ }^{1}$, \\ Melody L. Mak-Jurkauskas ${ }^{2}$, Jagadishwar R. Sirigiri ${ }^{3}$, Patrick C. A. van der Wel ${ }^{1}$, Judith \\ Herzfeld $^{2}$, Richard J. Temkin ${ }^{3}$, and Robert G. Griffin 1 ,a) \\ ${ }^{1}$ Department of Chemistry and Francis Bitter Magnet Laboratory, Massachusetts Institute of \\ Technology, Cambridge, Massachusetts 02139, USA \\ ${ }^{2}$ Department of Chemistry, Brandels University, Waltham, Massachusetts 02454, USA \\ ${ }^{3}$ Plasma Science and Fusion Center, Massachusetts Institute of Technology, Cambridge, \\ Massachusetts 02139, USA
}

\section{Abstract}

Dynamic nuclear polarization (DNP) is a method that permits NMR signal intensities of solids and liquids to be enhanced significantly, and is therefore potentially an important tool in structural and mechanistic studies of biologically relevant molecules. During a DNP experiment, the large polarization of an exogeneous or endogeneous unpaired electron is transferred to the nuclei of interest (I) by microwave $(\mu \mathrm{w})$ irradiation of the sample. The maximum theoretical enhancement achievable is given by the gyromagnetic ratios $\left(\gamma_{e} / \gamma_{l}\right)$, being $\sim 660$ for protons. In the early 1950s, the DNP phenomenon was demonstrated experimentally, and intensively investigated in the following four decades, primarily at low magnetic fields. This review focuses on recent developments in the field of DNP with a special emphasis on work done at high magnetic fields ( $\geq 5 \mathrm{~T})$, the regime where contemporary NMR experiments are performed. After a brief historical survey, we present a review of the classical continuous wave (cw) DNP mechanisms-the Overhauser effect, the solid effect, the cross effect, and thermal mixing. A special section is devoted to the theory of coherent polarization transfer mechanisms, since they are potentially more efficient at high fields than classical polarization schemes. The implementation of DNP at high magnetic fields has required the development and improvement of new and existing instrumentation. Therefore, we also review some recent developments in $\mu \mathrm{w}$ and probe technology, followed by an overview of DNP applications in biological solids and liquids. Finally, we outline some possible areas for future developments.

\section{Introduction/Background}

In 1953 Overhauser proposed that the large Boltzmann polarization of unpaired electrons could be transferred to neighboring nuclei by saturating the corresponding electron paramagnetic resonance (EPR) transition. The result of this process would be an enhancement of the signal intensities of the associated nuclear magnetic resonance (NMR) signals by a factor of $\left(\gamma_{e} / \gamma_{l}\right) \sim$ 660 in the case of protons. ${ }^{1}$ The validity of this suggestion was vigorously debated until Carver and Slichter reported an experiment in which they polarized Li (Ref. 2 ) and later ${ }^{23} \mathrm{Na}$ and ${ }^{1} \mathrm{H}$ nuclei, ${ }^{3}$ achieving signal enhancements of $\sim 100$. This experiment, now known as the Overhauser effect (OE) and widely used in solution NMR experiments for measuring ${ }^{1} \mathrm{H}-{ }^{1} \mathrm{H}$ distances, was the first demonstration of dynamic nuclear polarization (DNP).

a)Author to whom correspondence should be addressed. rgg@mit.edu. Phone: +1 617-253-5597. FAX: +1 617-253-5404. URL: http://web.mit.edu/fbml/cmr/griffin-group/index.html. 
In the following decade, additional pioneering DNP experiments were performed in both solids and liquids. In 1958 the solid effect (SE) was discovered, which required the use of polarizing agents with a homogeneous EPR linewidth $(\delta)$ and an inhomogeneous spectral breadth $(\Delta)$ smaller than the nuclear Larmor frequency $\left(\delta, \Delta<\omega_{0 I}\right){ }^{4,5}$ Subsequently, Kessenikh et al. $, 6,7$ Hwang and Hill, ${ }^{8,9}$ and Wollan ${ }^{10}$ reported $9 \mathrm{GHz}$ DNP experiments on systems with inhomogeneously broadened EPR spectra. The polarization mechanism in this case is the cross effect (CE), which is the dominant continuous wave (cw) mechanism at high magnetic fields. Specifically, the CE is operative when the polarizing agent has an inhomogeneous broadened EPR spectrum whose breadth $\Delta$ is larger than the nuclear Larmor frequency $\omega_{0 I}$, and, concurrently, the homogeneous linewidth $\delta$ remains small $\left(\Delta>\omega_{0 I}>\delta\right)$. The third regime, where the homogeneous EPR linewidth is larger than the nuclear Larmor frequency $\left(\delta>\omega_{0 I}\right)$, is referred to as thermal mixing ${ }^{11}$ (TM) and requires the existence of a high concentration of paramagnets.

There is currently a renaissance occurring in the development and application of DNP driven by the desire of the spectroscopist to enhance signal intensities in NMR spectra of both solids and liquids. This renaissance began in the 1980s with the appearance of magic angle spinning (MAS) experiments devoted to solids where the necessity of observing low- $\gamma$ nuclei $\left({ }^{13} \mathrm{C},{ }^{15} \mathrm{~N}\right.$, etc.) limits the sensitivity of the experiment. Thus, MAS-DNP methodology was developed to enhance sensitivity in ${ }^{13} \mathrm{C}$ spectra of polymers, carbonaceous, and other materials. These experiments were constrained to low fields $\left(\leq 60 \mathrm{MHz}\right.$ for $\left.{ }^{1} \mathrm{H}\right)$ because of the limited frequency range of klystron sources $(\leq 40 \mathrm{GHz})$ used to produce microwaves. At the same time, solid-state NMR was reaping tremendous benefits from the availability of superconducting magnets operating at fields of $\geq 5 \mathrm{~T}\left(\sim 200 \mathrm{MHz}\right.$ for $\left.{ }^{1} \mathrm{H}\right)$, which yields significant improvements in sensitivity and spectral resolution. Thus, today it is not uncommon to record MAS spectra at $16-21 \mathrm{~T}\left(700-900 \mathrm{MHz}\right.$ for $\left.{ }^{1} \mathrm{H}\right)$. The success of these high-field MAS experiments stimulated interest in developing DNP at high frequencies, and this in turn motivated innovations in a number of areas required to buoy the experiments to fruition: technological developments in high-frequency microwave $(\mu \mathrm{w})$ sources, transmission lines, and low temperature, multiple resonance MAS NMR probes. In addition, there have been important advances in the design of paramagnetic polarizing agents required for DNP. This in turn has enabled MAS experiments on membrane and amyloid proteins that are either difficult or impossible because of limited signal-to-noise. Therefore, high-frequency DNP is yet another example where the development of new instrumentation and methodology enables new areas of scientific inquiry.

In this overview, we focus primarily on the principles and applications of DNP MAS experiments at high magnetic fields and $\mu \mathrm{w}$ frequencies, which we arbitrarily define as $\geq 5 \mathrm{~T}$ corresponding to $\mu \mathrm{w}$ irradiation at frequencies $\geq 140 \mathrm{GHz}$ (for $g \sim 2$ ). For technical reasons discussed below, $\mu \mathrm{w}$ power levels in the range of $\sim 10 \mathrm{~W}$ are desirable. One $\mu \mathrm{w}$ source that is capable of operating in this frequency regime and producing this level of output power is the gyrotron, a cyclotron resonance maser, and accordingly, we introduced this source into DNP experiments in the early 1990s initially at $140 \mathrm{GHz}($ Ref. 12$)$ and more recently at $250 \mathrm{GHz}$ (Ref. 13 ) and $460 \mathrm{GHz} .{ }^{14}$

In addition, gyrotron technology can also be used to perform pulsed EPR experiments at high $\mu \mathrm{w}$ frequencies, ${ }^{15}$ which is of particular interest since pulsed DNP schemes potentially do not suffer from the unfavorable field dependence of the classical DNP mechanisms (SE and CE/ TM). Several different approaches to time domain experiments—nuclear spin orientation via electron spin locking (NOVEL), ${ }^{6}$ the integrated solid effect (ISE), ${ }^{17,18}$ dressed state solid effect $^{19}$ (DSSE) and rotating frame DNP (RF-DNP) (Refs. 68-70) - have set the stage for future developments in this area of DNP methodology. Because of the availability of gyrotron technology, as well as other innovations, the future of high-field DNP appears very promising. 
Although the focus of our efforts is high-field solid-state DNP, we also discuss recent experiments on liquids. Solution-state DNP experiments were first reported by Bennett and Torre ${ }^{20}$ and liquids were extensively studied in the early work of Hausser et al. ${ }^{21}$ who used the $\mathrm{OE}$ to transfer electron polarization to ${ }^{13} \mathrm{C},{ }^{19} \mathrm{~F}$, and ${ }^{31} \mathrm{P}$ nuclei. Further work was done by Mueller-Warmuth et al. ${ }^{22}$ who focused on the influence of molecular motion on the electron OE. More recent efforts to polarize liquids have utilized flow techniques ${ }^{23,24}$ or approaches where the sample is polarized as a solid at low temperatures and dissolved or melted and the liquid-state spectrum observed. ${ }^{25,26}$ Finally, we note that high nuclear spin polarizations can also be created by several other methods such as polarization transfer from optically excited states or polarized noble gases. For example, the high spin polarization in para-hydrogen can be used to enhance nuclear polarization in a chemically induced DNP (CIDNP) experiment.

${ }^{27}$ Another possibility is the use of hyperpolarized xenon in a spin polarization induced nuclear Overhausser effect (SPINOE) experiment described by Bifone et al. ${ }^{28}$ More recently, it was shown that caged hyperpolarized xenon can be used as a biosensor in magnetic resonance imaging (MRI) experiments. ${ }^{29}$ The discovery of photochemically induced dynamic electron polarization in chloroplasts ${ }^{30}$ resulted in a new class of experiments that utilize photochemically induced dynamic nuclear polarization (photo-CIDNP). ${ }^{31}$ For example, the observation of photo-CIDNP by solution NMR led to the formulation of the radical pair mechanism $^{32}$ and later to photo-CIDNP in solid-state NMR (ssNMR) MAS spectra. ${ }^{33}$

An effect similar to the SE can be used to transfer polarization between an excited electronic triplet state and a nucleus via forbidden transitions. This mechanism is referred to as microwave induced optical nuclear polarization and was introduced by Deimling et al. ${ }^{34}$ Other polarization schemes rely on the use of ${ }^{3} \mathrm{He}\left({ }^{\text {Ref. }}{ }^{35}\right.$ ) and quantum dots. ${ }^{36-38}$ These techniques, however, are of a much more specific interest and are beyond the scope of this review.

The remainder of this overview is organized as follows. In Sec. II we discuss cw and time domain polarization mechanisms, and in Sec. III we describe polarizing agents designed to be used in high-field experiments. Section IV is devoted to the most recent developments in $\mu \mathrm{w}$ technology, low temperature probe technology, and other instrumentation that enables DNP. We view these instrumental developments as very important since it is presently progress in this area more than anything else that limits applications of DNP. Some recent successful applications of DNP are described in Sec. V and we close in Section VI with a summary of prospective developments.

There are a number of excellent reviews on solid-state DNP by Jeffries, ${ }^{39}$ Abragam and Goldman, ${ }^{40,41}$ Atsarkin, ${ }^{42}$ and Wind et al. ${ }^{11}$ and liquid-state experiments are discussed by Hausser et al. ${ }^{21}$ and Mueller-Warmuth et al. ${ }^{22}$ Although these reviews focus on the early literature of the field, they are well written and contain much information that is currently useful.

\section{Polarizing Mechanisms in DNP Experiments}

To understand the polarization transfer mechanisms operative in a DNP experiment, we consider the general static Hamiltonian for an electron-nuclear system of the form

$$
\begin{aligned}
H & =H_{S}+H_{I}+H_{S I}=\omega_{0 s} S_{Z}-\omega_{01} I_{Z}+H_{S I}^{\mathrm{iso}}+H_{S I}^{\mathrm{dip}} \\
& =\omega_{0 S} S_{Z}-\omega_{01} I_{Z}+A\left(S_{Z} I_{Z}+S_{Y} I_{Y}+S_{X} I_{X}\right)+B S_{X} I_{Z},
\end{aligned}
$$

where $\omega_{0 S}$ and $\omega_{O I}$ are the electron and nuclear Larmor frequencies, respectively, $S_{i}$ and $I_{i}$ are the electron and nuclear spin operators, $H_{S I}^{\text {iso }}$ is the isotropic (Fermi contact) hyperfine 
interaction between the electron and the nucleus, and $H_{S I}^{\mathrm{dip}}$ is the anisotropic dipolar coupling. The hyperfine coupling $H_{S I}$ is given in a basis where the coefficients $A$ and $B$ denote the secular and pseudosecular hyperfine interactions. Note that this convention is the reverse of that commonly employed in ssNMR literature where $I$ is usually $\mathrm{H}$ and $S$ is ${ }^{13} \mathrm{C},{ }^{15} \mathrm{~N}$. The energy levels that result from this Hamiltonian are shown in Fig. 1(a).

Several excellent monographs covering the quantum mechanical treatment of electron-nuclear interactions under the influence of $\mu \mathrm{w}$ irradiation are available ${ }^{43-48}$ and the interested reader is referred to literature.

\section{A. Continuous wave DNP polarizing schemes}

There are presently four cw DNP mechanisms that have been successfully applied to solids and liquids- the $\mathrm{OE},{ }^{1}$ the $\mathrm{SE},{ }^{4,5,49}$ the $\mathrm{CE},{ }^{6,8,9}$ and $\mathrm{TM} .{ }^{41}$ In particular, the OE has also been used extensively in studies of liquids. Most of the applications of these DNP mechanisms have been performed at low magnetic fields, and the theoretical treatments published in the literature reflect this fact. In this section we present a brief overview for each mechanism with an emphasis on high-field theory when available.

1. Overhauser effect-The OE is a DNP mechanism which requires the presence of relaxation processes that simultaneously flip an electron and a nuclear spin. In liquids, these relaxation processes are based on time-dependent dipolar and scalar interactions between the electrons and the nuclei, which are governed by molecular rotational and translational motion in the dipolar case or chemical exchange and/or fast relaxation of one of the spins in the scalar case.$^{50}$ In solids, the OE requires the presence of mobile electrons such as the electrons in the conduction band of a metal ${ }^{2}$ one-dimensional (1D) organic conductors such as fluoranthene. ${ }^{51}$ The success of the OE depends on the condition $\omega_{0 S} \tau<1$ with $\tau$ the rotational correlation time of the paramagnetic species. This condition is difficult to satisfy when $\omega_{0 S}$ becomes large. Thus, the efficiency of the OE decreases at high magnetic fields, even though it likely does not go to zero. ${ }^{52}$ However, the $\mathrm{OE}$ is presently the only practical mechanism for the direct polarization of liquids. The following section outlines the main features of the OE, while a more detailed description can be found in the reviews of Hausser et al. ${ }^{21}$ and Mueller-Warmuth et al. ${ }^{22}$ and in the recent publication by Hoefer et al. ${ }^{52}$

The energy level diagram describing the transitions responsible for the $\mathrm{OE}$ is shown in Fig. 1 (a). The rates of the zero- and double-quantum transitions are $W_{0}$ and $W_{2}$, respectively, while the rates for the EPR transitions are denoted as $W_{S}$ and the corresponding rate for the NMR transitions is $W_{I} . W^{0}$ is the nuclear relaxation rate in the absence of electrons. In the OE, the allowed EPR transitions are saturated, while the zero- and double-quantum transitions induce changes in the nuclear spin population resulting in the DNP effect, with the observed enhancement defined as $\varepsilon=\left\langle I_{Z}\right\rangle /\left\langle I_{\mathrm{eq}}\right\rangle$. A rigorous treatment, which involves solving the rate equations for this system, leads to the following equation for the enhancement: ${ }^{21}$

$$
\varepsilon=1-\rho f S \frac{\gamma_{S}}{\gamma_{I}}
$$

with

$$
\rho=\frac{W_{2}-W_{0}}{W_{0}+2 W_{I}+W_{2}}
$$




$$
\begin{gathered}
f=\frac{W_{0}+2 W_{I}+W_{2}}{W_{0}+2 W_{I}+W_{2}+W^{0}}, \\
s=\frac{\left\langle S_{0}\right\rangle-\left\langle S_{Z}\right\rangle}{\left\langle S_{0}\right\rangle} .
\end{gathered}
$$

The coupling parameter $\rho$ depends on the relative contributions of the scalar and dipolar couplings and can range from -1.0 (pure scalar coupling) to 0.5 (pure dipolar coupling). Therefore, the maximum enhancement in a liquid sample, where the dipolar coupling governs the relaxation, is $\sim 330$, whereas in $1 \mathrm{D}$ conductors an enhancement of 530 was observed, ${ }^{51}$ which is very close to the theoretical value of 660 .

The couplings on the other hand depend on the electron Larmor frequency $\omega_{0 S}$ and the degree of molecular motion manifested in the correlation time $\tau$. This dependence is plotted in Fig. 2, which illustrates that the process is inefficient at high magnetic fields. The experimental aspects of determining $\rho$ are discussed in the articles of Hausser et al. ${ }^{21}$ Armstrong and Han, ${ }^{53}$ Grucker et al. ${ }^{54}$ and more recently by Hoefer et al. ${ }^{52}$ he leakage factor $f$ describes the nuclear spin relaxation by the electron spins and can assume a value between 0 (no relaxation due to the electron-nuclear coupling) and 1 (no other relaxation mechanisms). When the electron transitions are saturated completely $\left(\left\langle S_{Z}\right\rangle=0\right)$, the saturation factor $s$ has a value of 1 , although the saturation achieved depends on the number of hyperfine lines in the spectrum. This is important if polarizing agents based on nitroxide radicals are used. Due to the large electron-nitrogen hyperfine coupling ( $\sim 50 \mathrm{MHz})$, the EPR spectrum shows a well-resolved doublet (for ${ }^{15} \mathrm{~N}, \mathrm{I}=\frac{1}{2}$ ) or triplet (for ${ }^{14} \mathrm{~N}, I=1$ ), as opposed to the trityl radical featuring only a single EPR line.

The contribution of the scalar coupling can attenuate the enhancement if there is a concurrent time-dependent spin exchange. This can be described by an additional factor $\beta$, which can vary between 0 (no contribution of the scalar coupling) and 1 (large contribution of the scalar coupling). This dependence is shown in Fig. 2 for a number of different values of $\beta$.

2. Solid effect-The solid effect is a two-spin process, which relies on the mixing of states caused by the nonsecular component $B$ of the hyperfine coupling. ${ }^{41}$ The Boltzmann population distribution at thermal equilibrium is shown in Fig. 1(b).

The pseudosecular term $B$ in the Hamiltonian contains terms of the form $S_{Z} I^{+}$and $S_{Z} I^{-}$that lead to a mixing of the states of the system. The new mixed states are $\left|1^{\prime}\right\rangle=|1\rangle+q|2\rangle, \mid$ $\left.2^{\prime}\right\rangle=|2\rangle-q|1\rangle,\left|3^{\prime}\right\rangle=|3\rangle-q|4\rangle$ and $\left|4^{\prime}\right\rangle=|4\rangle+q|3\rangle$. The coefficient $q$ can be calculated by first order perturbation theory and is given by ${ }^{11}$

$$
q=-\frac{3}{4} \frac{\gamma_{s} \gamma_{I}}{\omega_{0 I}} \frac{1}{r^{3}} \sin \theta \cos \theta e^{-i \phi},
$$

where $r, \theta$, and $\varphi$ are the polar coordinates describing the electron-nuclear vector. Irradiation at $\omega_{0 S}+\omega_{0 I}$ or $\omega_{0 S}-\omega_{0 I}$ leads to zero-quantum [Fig. 1(c)] or double-quantum transitions [Fig. 1(d)], the probability of which is proportional to $4 q^{2} \cdot{ }^{41}$ Since $q$ is proportional to $\omega_{0 I}$, the 
transition probability and the enhancement scale with $\omega_{0 !}^{-2}$. This field dependence has thus far restricted the utility of the SE in high-field DNP experiments.

Another issues of a practical nature which affects the applicability of the SE is the fact that it requires a polarizing agent with a relatively narrow EPR spectrum. In particular, the homogeneous width $\delta$ and the inhomogeneous breadth $\Delta$ of the EPR spectrum have to be much smaller than $\omega_{0 I}$ to ensure that only one of the forbidden transitions is excited at a time. Simultaneous irradiation of both transitions leads to partial or complete cancellation of the polarization effect caused by each transition, a situation known as the differential solid effect (DSE). ${ }^{17}$

3. Cross effect/thermal mixing-The CE was first described in the 1960s by Kessenikh et al.,${ }^{6,7}$ a few years later by Hwang and Hill ${ }^{8,9}$ and then by Wollan. ${ }^{10}$ Unlike the SE, this new mechanism was based on allowed transitions and involved the interaction of electron spin packets in an inhomogeneously broadened EPR line. Treatments of a similar effect, called thermal mixing, in a homogeneously broadened EPR line appeared a few years later. ${ }^{11,55-}$

57 The intermediate case of an EPR line broadened by both homogeneous and inhomohogeneous interactions was treated by Wollan. ${ }^{10}$ This early theoretical work considers the CE/TM mechanisms at low magnetic field only, and it is only recently that the CE and TM have been used for DNP at high magnetic fields. ${ }^{15,58-61}$ Although they are the most efficient cw irradiation mechanisms known today, the theoretical framework describing them is not yet complete.

At high magnetic fields, the CE is defined as a three-spin process, which involves the interaction between two dipolar coupled electrons with EPR frequencies $\omega_{0 S 1}$ (electron spin 1) and $\omega_{0 S 2}\left(\right.$ electron spin 2 ) that satisfy the relation ${ }^{60}$

$$
\omega_{0 S 2}-\omega_{S 01}=\omega_{01}
$$

This is the dominant mechanism when the EPR line is inhomogeneously broadened by the $g$ anisotropy and the electrons are weakly coupled via electronic cross relaxation.

The CE requires that the inhomogeneous breadth $\Delta$ of the EPR spectrum is larger than the nuclear Larmor frequency $\omega_{0 I}$ to satisfy the condition that there are two molecular orientations, resulting in two effective EPR resonance frequencies, separated by the correct frequency. At the same time, the homogeneous width must satisfy the condition $\delta<\omega_{0 I}$. This condition is achieved when biradicals are used as polarizing agents, where the presence of a dipolar coupling between the two electrons can dramatically improve the efficiency of the DNP effect. In contrast, the TM mechanism involves a homogeneously broadened EPR line arising from multiple dipolar coupled electrons. Here, the condition $\delta>\omega_{0 I}$ is satisfied. This condition implies that at high magnetic fields the concentration of the polarizing agent must be high, which could unfortunately compromise the resolution in a MAS NMR experiment.

The thermal equilibrium spin population for a three-spin system is shown in Fig. 3(a). Note that there is no degeneracy present in the general case. However, when the appropriate polarizing agent is used, i.e., when there are two dipolar coupled electrons separated in frequency by $\omega_{0 I}$, levels $|4\rangle$ and $|5\rangle$ or $|6\rangle$ and $|3\rangle$ become degenerated [Figs. 3(b) and 3 (c)]. Irradiation of $\omega_{0 S 1}$ saturates the EPR transitions for one of the two dipolar coupled electrons, and the $\mathrm{CE}$ transitions $\left(\omega_{\mathrm{CE}}\right)$ ensure that there is a negative enhancement of the nuclear polarization [Fig. 3(b)]. Similarly, irradiation of the other EPR transition $\left(\omega_{0 S 2}\right)$ leads to positive enhancement [Figure 3(c)]. 
Since the linewidth of the EPR spectrum scales with the magnetic field, the degeneracy condition becomes more difficult to satisfy at higher magnetic fields. As a result, enhancements scale as $\mathrm{B}_{0}^{-1}$. Despite this unfavorable field dependence, the CE/TM mechanisms have been used successfully to polarize biological solids at high magnetic fields. ${ }^{62,63}$

The TM effect is usually treated from a thermodynamic point of view, based on the concept of spin temperature. ${ }^{55}$ The electron-nuclear spin system can be described as a set of three interacting baths, each characterized by a spin temperature: the electron Zeeman system (EZS), the electron dipolar system (EDS), and the nuclear Zeeman system (NZS). Off resonance irradiation of the allowed EPR transition results in a large polarization gradient across the EPR line, which is equivalent to cooling the EDS. This bath is in thermal contact with the NZS, which is also cooled in an energy-conserving three-spin electron-electron-nuclear exchange process, leading to DNP enhancement. The TM process can be direct, i.e., the enhancement is caused by the direct coupling between the NZS and the EDS, or indirect, when both allowed and forbidden transitions are induced. ${ }^{11}$ However, compared to the CE, the TM effect is less efficient and results in smaller enhancements.

\section{B. Pulsed DNP}

In principle, pulsed DNP experiments based on coherent polarization transfer such as the Hartmann-Hahn cross polarization (HHCP) are more efficient and do not show a field dependence of the polarization transfer with increasing magnetic field strengths. ${ }^{64}$

Currently, HHCP is an essential pillar in the standard repertoire of techniques in ssNMR spectroscopy, where the large polarization of one nucleus (e.g., $\left.{ }^{1} \mathrm{H}\right)$ is transferred to another nucleus $\left(\mathrm{e} . \mathrm{g} .,{ }^{13} \mathrm{C},{ }^{15} \mathrm{~N}\right) .{ }^{65,66}$ This requires irradiating both nuclei with rf fields, and the rotating frame Hartmann-Hahn matching condition, given by

$$
\omega_{11}=\omega_{1 S},
$$

has to be satisfied. In Eq. (8), $\omega_{1 I / S}$ is the field strength of the applied rf field and $I$ and $S$ denote two different spins. In ssNMR experiments involving spin- $\frac{1}{2}$ nuclei, the rf fields are sufficiently strong that they dominate all other interactions. This permits the excitation of the entire NMR spectrum and efficient spinlocking of both spin species. Therefore, the HartmanHahn condition [Eq. (8)] can be easily fulfilled.

In contrast, the $g$ anisotropy and hyperfine interactions can lead to large inhomogeneous broadening of high-field EPR spectra, and their spectral breadth $\Delta$ can easily exceed several hundred megahertz. Furthermore, usually, the $\mu \mathrm{w}$ field strengths are one to two orders of magnitude smaller. As a result, the matching condition in a electron-nuclear cross-polarization (eNCP) experiment cannot be achieved by simply adjusting the $\mu \mathrm{w}\left(\omega_{1 e}\right)$ and rf field strengths $\left(\omega_{1 I}\right)$. Thus, off resonance effects of the electron spin must be considered in analyzing the problem.

Another substantial difference with the HHCP scheme used in NMR is related to the relative concentrations of both spin types. NMR-CP experiments are typically performed by transferring polarization from a network of abundant high- $\gamma$ spins $I$ to dilute low- $\gamma$ spins $S$. Thermodynamic arguments suggest ${ }^{67}$ that efficient CP transfer in a single thermal contact between the $I$ and $S$ spins is possible only if $N_{I} \gg N_{S}$, with $N_{I}$ and $N_{S}$ the spin concentrations of the $I$ and $S$ spins, respectively. In this case, the signal enhancement in HHCP of the $S$ spins is given by ${ }^{67}$ 


$$
\varepsilon=\left(\frac{\gamma_{I}}{\gamma_{S}}\right) \frac{1}{1+N_{S} / N_{I}} \stackrel{N_{I} \gg N_{S}}{\approx} \frac{\gamma_{I}}{\gamma_{S}},
$$

and the full ratio $\gamma_{I} / \gamma_{S}$ can be transferred. However, in eNCP the concentration of unpaired electrons from the polarizing agent (the high- $\gamma$ spins) is typically four orders of magnitude lower than the ${ }^{1} \mathrm{H}$ concentration. This leads to an enhancement of $\varepsilon<1$ and only a small fraction of the $\mathrm{H}$ polarization can be transferred in a single CP step. Fortunately, the much shorter electron relaxation time $T_{1 e}(\sim \mathrm{ms})$ allows for multiple $\mathrm{CP}$ contacts within the nuclear relaxation time $T_{l n}(\sim \mathrm{s}$, pumping of polarization). This argument is also valid for cw DNP mechanisms such as the SE or TM/CE where a low concentration of electrons is used to polarize a much larger population of nuclear spins.

Pulsed DNP schemes can be divided into two groups: (1) those which involve both coherent $\mu \mathrm{w}$ pulses and $\mathrm{cw}$ irradiation and (2) schemes which are based purely on coherent $\mu \mathrm{w}$ pulses.

\section{DNP based on pulsed and $\mathrm{Cw} \mu \mathrm{w}$ irradiation}

Rotating frame DNP(RF-DNP): The idea upon which rotating frame DNP is based originated with experiments performed by Bloembergen and Sorokin on a single crystal of $\mathrm{CsBr}$. In this case, the transverse Cs magnetization was developed by cooling the rare ${ }^{133} \mathrm{Cs}$ spins in the rotating frame with a ${ }^{133} \mathrm{Cs}$ spin lock pulse, while simultaneously irradiating the abundant ${ }^{79} \mathrm{Br}$ spin with a rf field of frequency $\omega=\omega_{\mathrm{Br}} \pm \gamma_{\mathrm{Cs}} B_{1, \mathrm{Cs}}{ }^{68}$

Wind et al. applied this approach to an electron-nuclear system at low magnetic fields ( $B_{0}=$ $\left.1.4 \mathrm{~T}, \omega_{0 S} / 2 \pi=40 \mathrm{GHz}\right) .{ }^{69,70}$ Subsequently, rotating frame DNP was shown to function at high magnetic fields $\left(B_{0}=5 \mathrm{~T}, \omega_{0 S} / 2 \pi=140 \mathrm{GHz}\right){ }^{71}$

In rotating frame DNP experiments, $\mathrm{CE}$ or $\mathrm{SE}$ experiments are performed in the nuclear rotating frame, where the transition probabilities of forbidden transitions are greatly enhanced. This results in more efficient polarization transfers from the electron spin system to the nuclear spin system with short electronic/nuclear irradiation times. Furthermore, no Hartmann-Hahn matching condition is required, which is often difficult to achieve for electron-nuclear spin systems due to the large mismatch in gyromagnetic ratios.

Integrated solid effect (ISE): As mentioned above, the SE becomes inefficient in the case $\delta \gg \omega_{0 I}$, since simultaneous saturation of the forbidden transitions at $\omega_{0 S} \pm \omega_{0 I}$ will cancel the polarization effect. However, if a forbidden EPR transition can be selectively inverted, prior to saturation of an allowed transition, the polarization effect can be recovered. This approach is known as the ISE, introduced by Henstra et al.. ${ }^{17,18}$

ISE experiments can be performed by inversion of the forbidden transition using a selective $\pi$ pulse, at a frequency $\omega_{0 S} \pm \omega_{0 I}$, followed by irradiation on resonance. A second possibility is to perform a magnetic field sweep through the entire EPR line, while simultaneously applying $\mathrm{cw}$ microwave irradiation at a constant frequency $\omega_{0 S}$. At sufficient $\mu \mathrm{w}$ power, each electron spin packet experiences an adiabatic fast passage, leading to an inversion of the electron spin polarization. Just before this inversion, the condition for the forbidden transition $\omega_{0 S} \pm \omega_{0 I}$ is fulfilled. This will be referred to as the adiabatic-ISE. To date, ISE experiments were performed at low magnetic fields $(9 \mathrm{GHz})$ using either electrons to polarize ${ }^{29} \mathrm{Si}$ nuclei or transferring the high polarization of a photoexcited triplet to surrounding protons. ${ }^{18,73,74}$ In Fig. 4 we show an ISE build up curve for the adiabatic-ISE. In this experiment the $\mathrm{Si}$ signal in p-type $\mathrm{Si}$ at $1.2 \mathrm{~K}$ is detected and a factor $\sim 20$ larger enhancement is obtained in an adiabatic-ISE as opposed to the DSE. 
However, both the ISE and the adiabatic-ISE have certain limitations. In particular, the ISE requires high-power $\mu \mathrm{w}$ pulses to excite a large number of spin packets, and the appropriate $\mu \mathrm{w}$ sources are currently not available at high $\mu \mathrm{w}$ frequencies. In contrast, the adiabatic-ISE requires a fast magnetic field sweep. Adiabatic field sweeps are easy to perform at low magnetic fields but technically more demanding at high magnetic fields, since superconducting sweep coil couples strongly to the main and shim coils and distort the homogeneity of the magnetic field required for high-resolution spectroscopy. However, despite these current limitations, the ISE is an appealing approach because of the large enhancements that can be achieved.

\section{DNP based on coherent pulses}

Nuclear spin orientation via electron spin locking: In a NOVEL experiment, the electron magnetization is locked in the electron rotating frame via an electron spin locking sequence. If the field strength of the locking pulse is adjusted such that $\omega_{1 S}=\omega_{0 I}$, the rotating frame/labframe Hartmann-Hahn condition is fulfilled and polarization can be transferred to the nuclei in the presence of a dipolar coupling. NOVEL was independently introduced by Henstra $e t$ $a l$. and Brunner et al. ${ }^{16,75}$ Figure 4 (bottom) shows the nuclear polarization buildup curve for a NOVEL experiment in which the electron polarization from pentacene guest molecules in a photoexcited triplet state is transferred to $\mathrm{H}$ of the naphthalene host crystal. An enhancement of 220 is achieved after a spin locking time $\tau_{L}<0.5 \mu \mathrm{s}$.

So far, NOVEL experiments have only been performed at $9 \mathrm{GHz} \mu \mathrm{w}$ frequencies, where highpower $\mu \mathrm{w}$ sources and amplifiers are available. ${ }^{72,74,76-78}$ At higher magnetic fields, and therefore higher nuclear Larmor frequencies, much higher $\mu \mathrm{w}$ field strengths are required. For example, at $5 \mathrm{~T}$ the ${ }^{1} \mathrm{H}$ Larmor frequency is $211 \mathrm{MHz}$, which must be matched by $\omega_{1 S}$. However, the current technology used in high-field EPR experiments, which is based on Gunn and impact ionization avalanche transit-time (IMPATT) diodes, produces $\mu \mathrm{w}$ field strengths of only a few megahertz. With the availability of high $\mu$ w power at high frequencies (e.g., provided by extended interaction klystrons ${ }^{79}$ and gyrotron amplifiers), this method may become more generally useful since it provides a simple polarization scheme without the requirement of additional rf pulses.

Dressed-state solid effect: The Dressed State SolidEffect experiment, introduced by Weis et al. ${ }^{19,80}$ is based on simultaneous, near resonant $\mu \mathrm{w}$ and rf irradiations. The selective excitation of dressed state transitions generates nuclear polarization in the nuclear laboratory frame on a time scale of tens of microseconds and polarization transfer to surrounding nuclei is possible even in the absence of nonsecular hyperfine coupling terms.

The DSSE is best understood in a doubly tilted rotating frame. Given the limited $\mu$ w power at high frequencies, the allowed EPR transitions are in general no longer excited with the same $\mu \mathrm{w}$ field strength resulting in unequal effective fields $\omega_{1 S}^{\alpha, \text { eff }}$ and $\omega_{1 S}^{\beta, \text { eff }}$ (see Fig. 5). As a result, the transformation into the electron (nuclear)-tilted frame is no longer possible with a single angle $\theta_{S / I}$ per spin type.

As Figure 5 shows, the conditions matched for CP include four effective fields. The general matching condition for the DSSE experiment is given by

$$
\begin{aligned}
& \sqrt{\left(\Omega_{S}+A / 2\right)^{2}+\omega_{1 S}^{2}}+\sqrt{\left(\Omega_{S}-A / 2\right)^{2}+\omega_{1 S}^{2}} \\
& = \pm\left[\sqrt{\left(\Omega_{I}+\tilde{A} / 2\right)^{2}+\tilde{\omega}_{1 I}^{2}}+\sqrt{\left(\Omega_{I}+\tilde{A} / 2\right)^{2}+\tilde{\omega}_{1 I}^{2}}\right]
\end{aligned}
$$


with the resonant offsets given by $\Omega_{S}$ and $\Omega_{I}$, the $\mu \mathrm{w}$ and rf field strengths given by $\omega_{1 S}$ and $\tilde{\omega}_{1 I}$, and the hyperfine coupling constant given by $A$ and $\tilde{A}$, respectively. While the electrons experience the full hyperfine coupling strength $A$, the nuclei are partially decoupled due to the $\mu \mathrm{w}$ irradiation resulting in a reduced hyperfine coupling $\tilde{A} .{ }^{81}$

As in NMR-CP, the polarization transfer is driven by the zero-quantum $\left(S^{+} I^{-}+S^{-} I^{+}\right)$or the double-quantum operator $\left(S^{+} I^{+}+S^{-} I^{-}\right)$. In the case of on resonance $\mu \mathrm{w}$ irradiation $\left(\Omega_{S}=0\right)$ but arbitrary hyperfine coupling, the matching condition is given by

$$
\Omega_{I} \approx \pm \sqrt{\omega_{1 S}^{2}+(A / 2)^{2}-\omega_{1 I}^{2}} \approx \pm \sqrt{\omega_{1 S}^{2}+(A / 2)^{2}}
$$

In this case, the width of the Hartmann-Hahn condition is determined by the distribution of hyperfine couplings and should allow for hyperfine selective polarization transfer. For $\left|\Omega_{I}\right| \approx$ $\omega_{1 S}$, only nuclei with weak couplings $A<\omega_{1 S}$ (bulk nuclei) are expected to be polarized.

Figure 6 shows an example of the high-field DSSE. The eNCP condition was monitored indirectly through the electron spin echo intensity. Two peaks dispersed symmetrically around the $\mathrm{H}$ Larmor frequency $\omega_{0 I}$ are observed at all $\mu \mathrm{w}$ power levels. The positions of the satellites are $\mu \mathrm{w}$ power dependent and shift towards $\omega_{0 I}$ when $\omega_{1 S}$ is reduced. The position of the symmetric peaks is in full agreement with the matching condition for weakly coupled nuclei [Eq. (11) for $A \rightarrow 0$ ] and on resonance $\mu \mathrm{w}$ irradiation. Furthermore, a change in rf power does not result in a detectable shift or broadening of the $\mathrm{CP}$ matching condition. However, a decrease in signal intensity is observed upon reduction of the rf power indicating that the polarization transfer is faster for larger rf fields (Fig. 6, upper trace).

\section{Polarizing Agents}

The mechanism governing the DNP experiment depends strongly on the choice of the polarizing agent, and is influenced by factors such as the width of the EPR spectrum, the radical solubility, the temperature dependence of relaxation times, etc. In addition, the polarizing agent can be either exogeneous (a mono- or biradical or metal ion added to the system) or, in principle, endogeneous (for example, a stable radical present in a protein). There are many examples of the use of exogeneous radicals as polarizing agents, and, to date, the optimal enhancements in MAS experiments have been achieved with biradicals dispersed in a glassy matrix. ${ }^{59,61} \mathrm{In}$ contrast only photo-CIDNP experiments have utilized endogeneous polarizing agents in solids. 82,83 In solution DNP, the polarizing agent can be dissolved in the solvent, ${ }^{50}$ attached to a support while the solution flows past it, ${ }^{24,84,85}$ or connected to a bulky particle such $\mathrm{Si}$ and suspended in solution. ${ }^{86}$

Five of the molecules that have been frequently used for polarizing agents in high-field DNP experiments are illustrated in Fig. 7. Two of these, trityl and bis- $\alpha, \gamma$-diphenylene- $\beta$-phenylallyl (BDPA), have either threefold or approximate threefold symmetry and yield EPR spectra that are relatively narrow. This makes them suitable for SE DNP experiments. TEMPO is a nitroxide based radical, which leads to the TM mechanism at sufficiently high concentrations, whereas its biradical derivatives, bis-TEMPO- $n$-ethylene oxide (BTnE) and 1-(TEMPO-4oxy)-3-(TEMPO-4-amino)-propan-2-ol (TOTAPOL) induce the CE DNP mechanism. These and similar nitroxide based free radicals/biradicals have been used for both liquid- and solidstate bio-DNP since they are stable, unreactive, and soluble in a wide variety of solvents. For example, TOTAPOL is soluble in glycerol/water solutions and is currently the polarizing agent of choice for experiments involving proteins. In addition to these $g \sim 2$ species, very successful 
DNP experiments have been performed with paramagnetic chromium (V) based compounds, which were used in the preparation of polarized targets for nuclear scattering experiments. ${ }^{87}$

\section{A. Polarizing agents for the SE}

The resolved SE requires that the homogeneous linewidth, $\delta$ of the radical is less than the nuclear Larmor frequency, $\omega_{0 I}$. This requirement ensures that the zero- and double-quantum forbidden transitions are not excited simultaneously and there is no cancellation of the positive and negative enhancement. Currently, only two radicals satisfy this property at high magnetic fields, BDPA ( ${ }^{\text {Ref. }}{ }^{88}$ ) and trityl. ${ }^{89,90}$ BDPA has an inhomogeneous linewidth of $\Delta \sim 20 \mathrm{MHz}$ at $211 \mathrm{MHz}{ }^{1} \mathrm{H}$ Larmor frequency and it was first used to study the $\mathrm{SE}$ in a polystyrene matrix. $11,12,56,91$ However, its utility for biological work is greatly limited by its insolubility in aqueous solutions. In contrast, the trityl radical, developed by Nycomed/Amersham was originally designed for low field OE imaging experiments..$^{90,92}$ It is very soluble in aqueous media, and because of its symmetry and the absence of large ${ }^{1} \mathrm{H}$ hyperfine couplings, it has a narrow EPR spectrum that supports the SE. Furthermore, trityl has been successfully used together with the HyperSense (Oxford Instruments, UK) to polarize aqueous solutions. ${ }^{25}$

Figure 8(a) shows the $140 \mathrm{GHz}$ EPR spectrum and the field dependent enhancement profile of trityl $(\Delta=42 \mathrm{MHz})$. The experimental data were obtained in a MAS experiment on ${ }^{13} \mathrm{C}$-urea dispersed in ${ }^{2} \mathrm{H}_{6}-\mathrm{DMSO} /{ }^{2} \mathrm{H}_{2} \mathrm{O} / \mathrm{H}_{2} \mathrm{O}=60: 34: 6(\mathrm{w} / \mathrm{w} / \mathrm{w})$ at $90 \mathrm{~K}$. The positive and negative enhancements correspond to the double- and zero-quantum SE transitions, which are clearly resolved. The maximum enhancement is $\sim 15$, which is relatively small but consistent with data obtained from BDPA and expected from a SE mechanism at high magnetic fields.

\section{B. Polarizing agents for TM and CE}

The TM and CE mechanisms are the dominant mechanisms when the breadth of the EPR spectrum is large compared to the nuclear Larmor frequency $\left(\Delta>\omega_{0 I}\right)$. If the EPR spectrum is homogeneously broadened, the TM governs the DNP process. The CE, on the other hand, is the dominant mechanism when the EPR spectrum is inhomogeneously broadened. Nitroxide based radicals/biradicals are very suitable for these mechanisms since their inhomogeneous EPR spectral width $\Delta$ is $\sim 600 \mathrm{MHz}$ at a ${ }^{1} \mathrm{H}$ Larmor frequency of $211 \mathrm{MHz}$.

Monomeric TEMPO at high concentrations exhibits partial homogeneous broadening in its EPR spectra, ${ }^{15}$ and DNP is thought to proceed partially through the TM mechanism. As illustrated in Fig. 8(b), the maximum and minimum enhancements observed are $\sim \pm 50$ at a magnetic field strength of $5 \mathrm{~T}(140 \mathrm{GHz})$ using a TEMPO concentration of $\geq 40 \mathrm{mM}{ }^{60}$ At a magnetic field strength of $9 \mathrm{~T}(250 \mathrm{GHz})$, the enhancement factor decreases to $\varepsilon \sim 20^{13}$. However, above this concentration, the electron-nuclear broadening becomes significant and limits the resolution in the spectra.

In contrast, the $\mathrm{CE}$ is the dominant mechanism when the intermolecular electron coupling is small and the intramolecular electron-electron dipole coupling is large ( $\sim 22 \mathrm{MHz}$ in the case of TOTAPOL). The biradical polarizing agents shown in Fig. 7 are typically used in CE DNP experiments at low concentrations $(\sim 10 \mathrm{mM})$. Thus, the CE operates in the limit $\Delta>\omega_{0 I}>\delta$, where strongly coupled electron spin packets are present that effectively communicate with one another and produce larger enhancements than those observed with monoradicals.

The first biradicals used for DNP belonged to the BTnE series and consisted of two TEMPO moieties connected through ehtylene glycol linkages. ${ }^{59}$ Depending on the length of the tether ( $n=2,3$, or 4), the electron-electron dipolar coupling increases from $\sim 11$ to $\sim 22 \mathrm{MHz}$, which is a significant improvement over the $\sim 0.3 \mathrm{MHz}$ intermolecular dipolar coupling observed in a $10 \mathrm{mM}$ solution of monomeric TEMPO. As a result, larger enhancements of $\varepsilon=175 \pm 25$ 
through the CE have been observed for the shortest member of the series $(n=2)$. The use of biradicals with stronger dipolar coupling and exhibiting greater enhancements also means that a lower concentration of the polarizing agent can be used, therefore reducing the residual paramagnetic broadening. Thus, while the enhancement obtained with BT2E increases by a factor of $\sim 4$, the overall electron concentration can be reduced by a similar factor from 40 to $10 \mathrm{mM}$.

The field dependent enhancement profile for BT2E is shown in Fig. 9(a) and it is slightly asymmetric with a positive maximum (175) that is larger than the minimum $(-150)$, which likely reflects the asymmetry in the EPR spectrum. Despite its success, the BTnE series is not sufficiently soluble in glycerol/water mixtures, which are the preferred medium for biological samples and they have been succeeded by TOTAPOL (see Fig. 7). ${ }^{61}$ This biradical consists of two TEMPO moieties tethered with a three-carbon chain, which leads to an average electronelectron dipolar coupling of $\sim 22 \mathrm{MHz}$. Although this biradical has a similar enhancement profile to BT2E [Fig. 9(a)], it offers the very important advantage of being soluble in aqueous media. This is achieved by the hydroxyl and secondary amine moieties on the tether and allows for its use in DNP experiments on biological systems.

The TOTAPOL tether, like the BT2E biradical tether, is conformationally flexible, even though the tether in TOTAPOL is shorter and more rigid (see Hu et al., this issue). However, the average orientation of the $g$ tensors of the two electrons appears to be improved yielding an enhancement extrapolated to infinite power of $\varepsilon^{\infty}=335 \pm 65$ while the corresponding number for BT2E is $\varepsilon^{\infty}=260 \pm 55$.

Figure 9(b) shows another interesting experimental result, which is the polarization buildup curve as a function of the $\mu \mathrm{w}$ irradiation time for a sample in a $2.5 \mathrm{~mm}$ (as opposed to a $4 \mathrm{~mm}$ ) rotor. The time constant characterizing the buildup curve is $\sim 9 \mathrm{~s}$, approximately the nuclear $T_{1}$ of the sample. Note, however, that the maximum enhancement achieved is 290 , which is much larger than the enhancement observed in a $4 \mathrm{~mm}$ rotor ( 170). This is most likely due to improved $\mu \mathrm{w}$ penetration into the sample.

Finally, we would like to point out a few strategies to further improve polarizing agents available for DNP. The ideal polarizing agent for the CE should have an EPR spectrum consisting of two narrow sharp lines separated by $\omega_{0 I}$ in order to satisfy the CE matching condition. ${ }^{10,60}$ Although such a polarizing agent has not yet been reported, Hu et al. proposed to approximate this situation by utilizing a narrow line radical (e.g., trityl or BDPA) tethered to a broad line radical (e.g., TEMPO). This idea can be validated with a physical mixture of trityl and TEMPO, ${ }^{60}$ where the separation between the trityl line and the $g_{y y}$ component of TEMPO is $225 \mathrm{MHz}$ at $5 \mathrm{~T}$, which closely matches the ${ }^{1} \mathrm{H}$ Larmor frequency of $211 \mathrm{MHz}$ [Fig. 8 (b)]. The observed enhancement is $\sim 160$, an improvement of a factor of 4 over the enhancement achieved with TEMPO alone [Fig. 8(c)]. Thus, biradicals utilizing this approach could offer even larger enhancements than are presently observed.

\section{Instrumentation}

The implementation of DNP experiments requires the addition of three pieces of instrumentation to existing NMR spectrometers-namely, a suitable $\mu \mathrm{w}$ source, a waveguide to transmit the microwaves from the source to the NMR probe, and a multiple frequency NMR probe (typically ${ }^{1} \mathrm{H},{ }^{13} \mathrm{C}$, and ${ }^{15} \mathrm{~N}$ ) with a waveguide to irradiate the sample. Furthermore, since DNP processes are most efficient at low temperatures, it is necessary to perform MAS at $<90$ $\mathrm{K}$, and for dissolution or melting experiments, a means must be present to dissolve or melt the sample. Finally, it is necessary to irradiate the EPR spectrum at the position that produces the maximum enhancement. This is conveniently done with either a sweep coil on the magnet or, 
in principle, by tuning the microwave source. We now discuss each of these topics in more detail.

\section{A. Microwave sources}

The $\mu \mathrm{w}$ power requirement for DNP depends strongly on the nature of the experiment to be performed. In DNP experiments on static samples a high $Q$ resonant $\mu \mathrm{w}$ cavity can be used since the sample size can be small compared to the wavelength of the radiation. However, the sizes of conventional MAS rotors $(2.5-4.00 \mathrm{~mm})$ used in experiments on biological systems are comparable to the wavelength of the $\mu \mathrm{w}(0.65-2.14 \mathrm{~mm})$. Thus, placing a rotor and a rf coil in a cavity significantly lowers its $Q$. Furthermore, the $\mu \mathrm{w}$ field strength $\omega_{1 S}$ is directly related to the available $\mu$ w power $P$ and the quality factor $Q_{\mu \mathrm{w}}$ by

$$
\omega_{1 S} \propto c \sqrt{Q_{\mu w} \cdot P}
$$

Therefore, high-power $\mu \mathrm{w}$ sources are required for DNP experiments without a resonance structure.

Several excellent reviews are available describing $\mu$ w sources, outlining the historical developments and giving future directions in $\mu \mathrm{w}$ technology. ${ }^{93-95}$ In general, two different types of $\mu \mathrm{w}$ sources can be distinguished: solid-state and vacuum electronic devices. Figure 10 provides an overview of high-frequency $\mu \mathrm{w}$ sources available currently. However, not all sources fulfill the requirements necessary for DNP or EPR applications. As we will see below, gyrotrons (cyclotron resonance masers) are high-frequency vacuum electronic devices that have many desirable features for EPR and DNP experiments.

1. Solid-state and other low power devices-Currently, the majority of high-frequency $\mu \mathrm{w}$ sources used in cw, low-power pulsed EPR spectrometers, and in some DNP experiments utilizing $\mu \mathrm{w}$ cavities, are solid-state devices. Typical examples include phase-locked Gunn diodes, IMPATT diodes or $\mathrm{CO}_{2}$ pumped far infrared (FIR) lasers. Phase-locked Gunn diodes provide stable $\mu \mathrm{w}$ irradiation of several tens of milliwatts. However, at higher $\mu \mathrm{w}$ frequencies (>200 GHz), the available output power drops dramatically (e.g., $\sim 5 \mathrm{~mW}$ at $400 \mathrm{GHz}$, Ref.

${ }^{96}$ ) and IMPATT devices assume a more important role as $\mu \mathrm{w}$ oscillators and amplifiers. They can operate in either $\mathrm{cw}$ or pulsed modes and deliver powers of up to $500 \mathrm{~mW}$ at $140 \mathrm{GHz}$. Despite their substantial complexity of operation, $\mathrm{CO}_{2}$ pumped FIR lasers have been used as low-power sources at series of discrete frequencies in the range of $160 \mathrm{GHz}-3 \mathrm{THz} .{ }^{96,97}$ However, DNP/MAS experiments that do not employ a resonant $\mu \mathrm{w}$ structure require several watts of $\mu \mathrm{w}$ power that is available from vacuum electronic devices. Therefore, solid-state sources are not applicable.

\section{Vacuum electronic devices}

General: Vacuum electronic devices are $\mu \mathrm{w}$ sources that are able to generate high $\mu \mathrm{w}$ power at high frequencies. Extended interaction oscillators (EIOs), extended interaction klystrons (EIKs), backward wave oscillators (BWOs), orotrons, and gyrotrons are several examples; some of them have been used successfully in EPR spectroscopy. EIOs and EIKs have been developed as remarkably powerful sources operating at 95 and $140 \mathrm{GHz}$ in $\mathrm{cw}$ and pulsed experiments. They are capable of delivering up to $1.2 \mathrm{~kW}$ peak power with pulse widths from a few nanoseconds to several microseconds. $.99,98$

BWOs (or carcinotrons) are $\mu \mathrm{w}$ devices operating in both $\mathrm{cw}$ and pulsed modes. At $100 \mathrm{GHz}$, they can generate more than $10 \mathrm{~W}$ in cw mode, and several kilowatts of pulsed power. However, 
the output power shows a strong frequency dependence and requires highly stabilized highvoltage power supplies. Furthermore, at higher frequencies, the available power drops dramatically ${ }^{93}$ (see Fig. 10).

Orotrons are nonrelativistic free-electron lasers, where the $\mu \mathrm{w}$ radiation is created when the electron beam passes close to the surface of a metallic grating and moves perpendicular to its periodic rulings. The incoherent and weak $\mu \mathrm{w}$ beam is transformed into coherent and intense radiation in a Fabry-Pérot resonator, and power levels up to $60 \mathrm{~mW}$ were achieved at a $\mu \mathrm{w}$ frequency of $360 \mathrm{GHz} .{ }^{99}$

The vacuum electronic devices described above are also called "slow-wave" devices. In particular, the $\mu \mathrm{w}$ irradiation is created by modulating an electron beam that passes close to a fragile interaction structure, whose size is on the order of the $\mu \mathrm{w}$ wavelength. As the power and frequency increase, so does the power density, and the lifetime of the slow-wave structure decreases since the interaction structure is easily destroyed. This significantly limits the lifetime of the slow-wave structure especially at high-power levels. Although there continue to be advances in technology, the current power levels and lifetimes of these devices limits their use to lower $\mu \mathrm{w}$ frequencies.

Gyrotons: Gyrotron oscillators and amplifiers are "fast-wave" devices that rely on a resonance phenomenon between the modes of an interaction structure and the electron beam in a magnetic field. The resonator can be overmoded and, as such, can have physical dimensions, which are much larger than the operating wavelength. This permits high peak and average power operation even at elevated frequencies without the risk of damage to the interaction structure. 100

In the frequency range of interest for DNP/NMR (presently $200-900 \mathrm{MHz}$ for ${ }^{1} \mathrm{H}$, corresponding to $140-590 \mathrm{GHz}$ for electrons), the gyrotron is the only device demonstrated capable of producing adequate power levels. There are now a number of excellent introductory books on gyrotron technology ${ }^{101-105}$ as well as several excellent review articles. ${ }^{100,106}$

A gyrotron is a vacuum electronic device that operates in a static magnetic field. It functions as an electron cyclotron resonance maser that emits coherent radiation near the relativistic electron cyclotron frequency, or its harmonics given by

$$
\omega_{c}=s \frac{e B_{0}}{\gamma \prime m c}
$$

with $B_{0}$ the magnetic field strength, $e$ the electron charge, $s$ the harmonics of the operational mode (integer), $m$ the electron mass, $c$ the speed of light, and $\gamma^{\prime}$ a relativistic mass factor (vide infra) given by

$$
\gamma /=\frac{1}{\sqrt{1-v^{2} / c^{2}}}
$$

with $v$ the velocity of the electron. When other experimental constraints are satisfied, the $\mu \mathrm{w}$ frequency $\omega_{c}$ is primarily determined by the strength of the magnetic field.

The physical layout of a gyrotron oscillator is shown in Fig. 11 (left). The compact design of the tube makes it compatible with operation in close proximity to a NMR spectrometer. 
Electrons, which are emitted from a cathode located in the electron gun [Fig. 11(1)], are accelerated by a high voltage (10-30 kV) applied between the anode and the cathode and in the presence of an external magnetic field they start to gyrate (rotate) about the magnetic field lines. While they move from the lower magnetic field region at the gun to the high-field region in the center of the magnet along the magnetic field lines, they increase their rotational energy. In the cavity region [Fig. 11(5)], a phenomenon referred to as bunching leads to the generation of microwaves (see Ref. 62 and references therein). The radiation excites a mode of the cavity, causing stimulated emission, which leads to high efficiency emission and output power. The microwaves are then extracted by a step-cut mode converter [Fig. 11(6)] through a cross bore in the magnet dewar and directed to the NMR sample through a waveguide. Finally, the remaining electron beam is collected in a water-cooled collector [Fig. 11(9)].

Currently, two gyrotrons are operating in DNP experiments at $140 \mathrm{GHz}$ (Refs. 12 and ${ }^{107}$ ) and $250 \mathrm{GHz}$ (Refs. 13 and ${ }^{62}$ ) and the application that they have enabled will be discussed below. A third device operating at $460 \mathrm{GHz}$ (corresponding to $700 \mathrm{MHz}$ for ${ }^{1} \mathrm{H}$ ) is already operational ${ }^{14,108}$ and its incorporation into a DNP experiment is under way. Figure 11 shows a photograph of the $460 \mathrm{GHz}$ gyrotron.

\section{B. Microwave waveguides}

Low-loss $\mu \mathrm{w}$ transmission lines are crucial for an efficient delivery of the $\mu \mathrm{w}$ irradiation. Unfortunately, fundamental waveguides become very inefficient with increasing $\mu \mathrm{w}$ frequencies (WR-08, >8 dB / m at $140 \mathrm{GHz}$ ). To minimize the loss, the $\mu \mathrm{w}$ irradiation can be delivered in an oversized waveguide (e.g., $K$ band, WR- $42,0.7 \mathrm{~dB} / \mathrm{m}$ at $140 \mathrm{GHz}$ ). Near the NMR coil (or cavity), the oversized waveguide is then tapered down to the fundamental waveguide. ${ }^{109}$ However, the overall loss will still be in the range of several decibels.

With a corrugated waveguide, operating in the circular $\mathrm{HE}_{11}$ mode, which has almost negligible Ohmic losses, the efficiency can be dramatically increased. ${ }^{110}$ No additional mode converters are necessary, since the gyrotron can be designed to deliver this mode. Corrugated waveguide transmission lines are well-established and currently used in high-frequency EPR as well as in DNP applications. ${ }^{62,111,112}$ For example, in the $250 \mathrm{GHz}$ DNP spectrometer, the overall loss of the transmission line is as low as $0.7 \mathrm{~dB} .{ }^{62}$ Furthermore, directional couplers are available to monitor the transmitted and reflected $\mu \mathrm{w}$ power. ${ }^{112}$

\section{Probes used for DNP experiments}

As mentioned above, DNP experiments on static samples and in some liquid-state experiments can utilize small $\mu \mathrm{w}$ cavities in combination with low-power sources. In contrast, DNP probes used for ssNMR MAS experiments cannot incorporate a $\mu$ w cavity, since the rotor containing the sample, and the NMR coil spoil the $Q$ of any cavity.

1. Probes with a resonant structure-In liquid-state DNP experiments, the sample size is kept very small to avoid excessive $\mu \mathrm{w}$ absorption by the solvent, especially if the dielectric constant of the solvent is high (e.g., water). At low $\mu$ w frequencies (e.g., $9 \mathrm{GHz}$ ) polarization in a DNP experiment can be performed in a standard $\mathrm{TE}_{102}$ rectangular EPR cavity. ${ }^{24,84} \mathrm{At}$ high frequencies, the cylindrical $\mathrm{TE}_{011}$ cavity first proposed by Grinberg et al. ${ }^{113}$ is commonly used in EPR experiments. However, the reduced size of the cylindrical resonator (e.g., $2.8 \mathrm{~mm}$ inner diameter at $140 \mathrm{GHz}$ ) does not allow a NMR rf coil to be mounted inside the resonator. An external rf coil in combination with a slotted cavity, commonly used in high-frequency electron nuclear double resonance (ENDOR), ${ }^{109,114}$ suffers from a dramatic loss of rf magnetic field strength at the sample position. A more suitable double-resonance design for DNP and ENDOR experiments is shown in Fig. 12 (left side) ${ }^{115}$ It was originally proposed by Hyde $^{116}$ and later used for ENDOR experiments at $X$-band frequencies. ${ }^{117}$ 
The metal wall, which serves as a cylindrical cavity operating in the $\mathrm{TE}_{011}$ mode, doubles as the cavity and rf coil. A fixed and a movable plunger with metal plates complete the cavity. Currently, this cavity operates at $140 \mathrm{GHz}$ (Ref. 115$)$ and features a double-tuned rf circuit for irradiation at ${ }^{1} \mathrm{H}$ and ${ }^{13} \mathrm{C}$ frequencies allowing rf field strengths of 100 and $50 \mathrm{kHz}$, respectively. The $\mu \mathrm{w}$ field strength $\omega_{1 S}$ is $2.5 \mathrm{MHz}$ at $15 \mathrm{~mW} \mu \mathrm{w}$ power. Using this cavity enhancement factors of 400 were achieved in a DNP experiment at low temperatures $(20 \mathrm{~K}) .{ }^{115}$ The cavity can also be used in liquid DNP experiments, since the electric field at the sample position is minimized.

2. Probes without a resonant structure-Historically, two different designs were used in DNP/MAS experiments. In the design featured by Schaefer and McKay, operating at 40 $\mathrm{GHz}$, the $\mu \mathrm{w}$ irradiation is applied along the rotor axis. ${ }^{91}$ The same design was later used at $140 \mathrm{GHz} .{ }^{109}$ However, the $\mu \mathrm{w}$ irradiation is highly attenuated parallel to the sample length, since the length of the rotor is a few centimeters. In current designs, the microwave irradiation occurs perpendicular to the rotor axis. ${ }^{119}$ A slightly modified design operating at $60 \mathrm{GHz}$ was recently introduced by Cho et al. ${ }^{120}$ while in the design of Wind et al. operating at $40 \mathrm{GHz}$, a horn-antenna/reflector combination was used. ${ }^{119}$ At higher frequencies (> $\left.140 \mathrm{GHz}\right)$, the microwaves are usually launched between the turns of the NMR coil due to space restrictions. Figure 12 (right) gives a schematic overview of a DNP probe for MAS-NMR experiments. A saphire rotor containing the sample (shown in purple) resides inside the MAS stator. The inner conductor of the transmission line doubles as a corrugated waveguide, and connects to a mitered, metal-mirrored assembly that terminates perpendicular to the rotor axis $1 \mathrm{~cm}$ from the sample. Since the $\mu \mathrm{w}$ are delivered as a Gaussian beam in a corrugated waveguide, the beam can be focused by carefully adjusting the miter bend on top of the probe to achieve optimum enhancements.

One major challenge of the rf circuit of a low temperature probe is to maintain the ability to tune the resonant circuit even at low temperatures. This can be done by locating all variable tuning elements outside the probe at room temperatures and delivering the rf power using a transmission line. ${ }^{121}$

Low temperature spinning is accomplished by using cold drive and bearing gas, and the excellent thermal conductivity of sapphire at low temperatures insures a uniform sample temperature. The gas is cooled in a heat exchanger and the cold gas is delivered by cryogenic transfer lines. ${ }^{122}$ Using nitrogen for spinning, temperatures as low as $\sim 85 \mathrm{~K}$ are achieved. To reach temperatures as low as $\sim 15 \mathrm{~K}$, helium gas is used. ${ }^{58,123,124}$

\section{Superconducting magnets}

For DNP experiments, the $\mu \mathrm{w}$ frequency or magnetic field strength is adjusted to maximize the enhancement. Since gyrotrons and other high-frequency $\mu \mathrm{w}$ devices operate at a fixed frequency, it is necessary to sweep the magnetic field. Furthermore, if the field profile of a polarizing agent needs to be recorded, the system should be equipped with a field-lock system. 125 In such a system, the NMR signal of a small liquid sample (e.g., water) is monitored, allowing the field determination to very high accuracy.

\section{Applications}

After the initial experiments demonstrating the ability to enhance nuclear spin polarization, DNP in solids largely became the province of high-energy physics. ${ }^{40}$ Thus, for many years, the main application of the technique was in experiments performed at low temperatures aimed at producing polarized targets used to investigate the spin dependence of nuclear forces in scattering experiments. These experiments continue today. 
A different application of DNP was stimulated by the introduction of Hartmann-Hahn cross polarization to high-resolution ssNMR. ${ }^{64,65}$ In this case DNP was used to enhance the polarization of ${ }^{19} \mathrm{~F}$ in $\mathrm{CaF}_{2}$, and this polarization was subsequently transferred to the rare ${ }^{43} \mathrm{Ca}$ spins for observation. ${ }^{126}$ This experiment was the predecessor to much of the research currently in progress.

In the 1980s DNP and MAS were integrated into experiments designed to enhance the ${ }^{13} \mathrm{C}$ signal in the studies of several samples including polymers, carbonaceous materials, diamond, etc. ${ }^{11,91,127}$ However, for reasons outlined above, essentially all of these applications were limited to low frequencies and mostly to experiments that demonstrated the ability to enhance signal-to-noise ratio. Here, we focus on some more recent applications to biological solids, liquids, and pulsed DNP.

\section{A. DNP applications to biological solids}

Hall et al. ${ }^{58}$ reported the first successful application of DNP to enhance ssNMR MAS spectra of biological macromolecules. In this experiment, a sample of ${ }^{15} \mathrm{~N}$-Ala-labeled T4 lysozyme was polarized and the DNP enhanced ${ }^{15} \mathrm{~N}$ solid-state CP/MAS spectra was recorded. An enhancement of $\sim 50$ was achieved for the alanine amide ${ }^{15} \mathrm{~N}$ signals. The experiments were performed in a frozen, glassy, aqueous solution of glycerol/water (60/40, w/w) using 4-aminoTEMPO as the polarizing agent. Aqueous glycerol solutions form a glass upon cooling and have been successfully used as cryoprotectants for biological samples at low temperatures. 128 Thereafter, the method was applied to record 1D spectra of other systems of biological interest—filamentous bacteriophages, purple membranes, ${ }^{129}$ and membrane and soluble proteins. ${ }^{130}$

Since the gyrotron provides a stable high-power source for $\mu \mathrm{w}$ irradiation, it is possible to integrate high-field DNP into state-of-the-art multidimensional ssNMR MAS experiments to obtain enhanced sensitivity. This was first demonstrated with two-dimensional (2D) high-field DNP enhanced correlation spectra of $\left[\mathrm{U}-{ }^{13} \mathrm{C},{ }^{15} \mathrm{~N}\right]$ proline at $140 \mathrm{GHz}\left(211 \mathrm{MHz}\right.$ for $\left.{ }^{1} \mathrm{H}\right)$ (Ref. $\left.{ }^{131}\right)$ and subsequently at $250 \mathrm{GHz}\left(380 \mathrm{MHz}\right.$ for $\left.{ }^{1} \mathrm{H}\right) .{ }^{13}$

More recent applications have focused on the areas of amyloid and membrane protein spectroscopy where ssNMR MAS experiments are the method of choice for structural studies. As illustrated in Fig. 13, high-field DNP can also be used to enhance NMR spectra of amyloidforming peptides. ${ }^{63}$ The samples consist of nanocrystals of the amyloid-forming peptide GNNQQNY $_{7-13}$, derived from the yeast prion protein Sup35p, dispersed in a glycerol/water matrix containing the biradical polarizing agent TOTAPOL. The crystals have an average width of 100-200 nm, and their known crystal structure ${ }^{132,133}$ suggests that the size of the biradical precludes its penetration into the crystal lattice. This was confirmed by the observation that $1 \mathrm{D}^{15} \mathrm{~N}$ spectra of the nanocrystals did not broaden even in the presence of high concentrations of polarizing agent (up to $50 \mathrm{mM}$ TOTAPOL, corresponding to $100 \mathrm{mM}$ electrons). Thus, polarization from the glycerol/water solvent diffuses over the dimensions of the crystal and results in an enhancement of the ${ }^{13} \mathrm{C}$ signals by a factor of $\sim 120$. The large signal enhancement facilitated the acquisition of a $2 \mathrm{D}^{13} \mathrm{C}-{ }^{13} \mathrm{C}$ homonuclear correlation spectrum, an experiment that would have been impossible in the absence of DNP. The spectrum, shown in Fig. 13, was acquired with $6 \mathrm{~ms}$ spin diffusion time and the total acquisition time was $20 \mathrm{~min}$. The linewidths of the peaks are likely governed by ${ }^{13} \mathrm{C}-{ }^{13} \mathrm{C} \mathrm{J}$ couplings and $n=0$ rotational resonance effects. ${ }^{134}$

More recently, $250 \mathrm{GHz}$ DNP was used to study bacteriorhodopsin (bR), a 248-residue integral membrane protein that functions as a light-driven ion pump..$^{62}$ The protein encapsulates an all-trans chromophore, which is attached to the protein via a Schiff base linkage lys 216 . To study a particular photocycle intermediate, the sample is irradiated with the optimal wavelength 
of light at a suitable low temperature. In the next step, the sample is cooled further to $\sim 90 \mathrm{~K}$ where the attenuated electronic and nuclear spin-lattice relaxation renders the polarization transfer more efficient.

Since the instrumentation is very stable, 2D spectra can be recorded over a period of several days. A typical 2D ${ }^{15} \mathrm{~N} /{ }^{13} \mathrm{C}$ correlation spectrum illustrating this point is shown in Fig. 14 . Because of the excellent signal-to-noise ratios, it was possible to observe cross peaks between the ${ }^{15} \mathrm{~N}$ Schiff base and C-15, C-14, C-13, and C-20 on the retinal and with $\mathrm{C} \varepsilon$ and $\mathrm{C} \delta$ of lys 216 . The spectrum in Fig. 14 was recorded in approximately $12 \mathrm{~h}$ and allowed the assignment of most retinal resonances in a single experiment. This would not have been possible without the factor of $\sim 90$ signal enhancement. Further experiments on the other photocycle intermediates of $\mathrm{bR}$ will be reported elsewhere.

\section{B. DNP in liquid phase}

1. Direct polarization-To date, the $\mathrm{OE}$ is the only mechanism available to directly polarize liquids. Since the OE becomes less efficient at higher magnetic field, one strategy to perform DNP on liquid samples is to polarize at low magnetic fields and perform the NMR experiment at high magnetic fields. To transfer the sample between high and low magnetic fields a flow system can be used.

In the late 1980s, the first flow experiments were carried out by Dorn and co-workers. Initially, the polarization and detection was performed in the same magnet, at a frequency of $9.3 \mathrm{GHz}$ $\left(0.35 \mathrm{~T}, 14 \mathrm{MHz}\right.$ for $\left.{ }^{1} \mathrm{H}\right)$ and enhancement factors for ${ }^{1} \mathrm{H}$ of up to $\varepsilon=-90$ were obtained. ${ }^{23}$,

${ }^{84}$ Later, the detection was performed at a magnetic field of $4.7 \mathrm{~T}\left(200 \mathrm{MHz}\right.$ for $\left.{ }^{1} \mathrm{H}\right)$ in a separate magnet ${ }^{85}$ yielding enhancement factors of $\varepsilon=-30$. In these experiments, the polarizing agent was either diluted in the sample (liquid-liquid intermolecular transfer, $\mathrm{L}^{2} \mathrm{IT}$ ) or immobilized on silica gel (solid-liquid intermolecular transfer, SLIT). The same technique was shown to yield large enhancements for ${ }^{13} \mathrm{C}$ (Refs. ${ }^{135}$ and ${ }^{136}$ ) and ${ }^{15} \mathrm{~N}$ (Ref. ${ }^{137}$ ) nuclei.

Recently, McCarney et al. used the SLIT experiment to polarize water for possible use in MRI experiments. ${ }^{24}$ These experiments showed that the hyperfine coupling of the nitroxide has to be taken into account for an accurate description of the Overhauser effect. ${ }^{53}$

2. Temperature jump experiments-A different strategy to obtain DNP enhanced spectra of molecules in solution is to perform the polarization at low temperatures in the frozen, solid state and then quickly melt the sample and record the liquid-state NMR spectrum. In this case, the enhancement, defined as $\varepsilon^{\dagger}$, is determined by the intensity of the DNP signal $\varepsilon$ at the irradiation temperature $\left(T_{\mu \mathrm{w}}\right)$ relative to the signal due to the Boltzmann polarization recorded at $300 \mathrm{~K}\left(T_{\mathrm{obs}}\right)$. Specifically since the polarization is generated at low temperature, an additional factor of $T_{\mathrm{obs}} / T_{\mu \mathrm{w}}$ has to be included in the calculation. Thus, $\varepsilon^{\dagger}$ is given by

$$
\varepsilon^{\dagger}=\varepsilon\left(T_{\mathrm{obs}} / T_{\mu w}\right)
$$

Currently, there are two experimental protocols that employ this approach—the "dissolution" experiment and the in situ temperature jump (TJ) experiment.

Dissolution experiment: The dissolution experiment was introduced by Ardenkjaer-Larsen et al. ${ }^{25}$ In this experiment, direct polarization of ${ }^{13} \mathrm{C}$ or ${ }^{15} \mathrm{~N}$ is performed at $1.2 \mathrm{~K}$ at a $\mu \mathrm{w}$ frequency of $94 \mathrm{GHz}$ using a $200 \mathrm{~mW}$ Gunn diode source. Furthermore, the EPR signal as well as relaxation data can be obtained by longitudinal detection ${ }^{138}$ in the apparatus used for dissolution experiments. 
Because of the low temperature, the low $\mu \mathrm{w}$ power, the long $T_{1 e}$ of the polarizing agent, and the fact that ${ }^{13} \mathrm{C}$ is polarized directly, the polarization time is very long ( $\geq 80 \mathrm{~min}$ ).

Subsequently, the frozen polarized sample is dissolved in superheated water. The sample is then shuttled to a NMR spectrometer, where the liquid-state NMR experiment is recorded. Enhancements of $\varepsilon=163\left(\varepsilon^{\dagger}=44400\right)$ for ${ }^{13} \mathrm{C}$ and $\varepsilon=86\left(\varepsilon^{\dagger}=23500\right)$ for ${ }^{15} \mathrm{~N}$ are reported for small molecules in a 1D experiment. Recently, the dissolution experiment was combinded with single-scan 2D NMR methods ${ }^{139}$ to record 2D NMR spectra of liquid within $\sim 0.1 \mathrm{~s} .{ }^{140}$

Although the dissolution experiment yields large values of $\varepsilon^{\dagger}$, this arises mostly from the Boltzmann temperature factor. In addition, the experiment has other limitations. First, the polarized sample is diluted by a factor of $\sim 150$ when melted. Second, since a direct polarization is performed rather than via the ${ }^{1} \mathrm{H}$ 's, it requires long periods. Third, because of the requirement of shuttling to a second magnet, it is not possible to rapidly repolarize the sample for further signal averaging and there is an inherent loss in the sample transfer. Because of the inability to recycle the experiment, many multidimensional liquid experiments are more difficult to implement, especially when the spectra are wide and the gradients required for single-scan experiments are large. However, the method has considerable potential as an analytical tool to study small molecules and may be useful in imaging experiments.

In situ TJ DNP experiment: The in situ TJ experiment introduced by Joo et al. ${ }^{26}$ is considerably different from the dissolution experiment. Here, the ${ }^{1} \mathrm{H}$ nuclei of the sample are polarized at low temperatures $(90 \mathrm{~K})$ using low concentrations of biradical polarizing agents. ${ }^{61}$ The ${ }^{1} \mathrm{H}$ polarization is then transferred to the low- $\gamma$ spins in a typical CP step and the sample is melted with an infrared laser pulse. The enhanced signal is observed in the presence of ${ }^{1} \mathrm{H}$ decoupling. This scheme and the respective pulse sequence is summarized in Fig. 15(a). Currently, this method yields enhancements on the order of $\varepsilon=133\left(\varepsilon^{\dagger}=400\right)$ for small molecules such as urea. The entire cycle can be repeated in situ and signal averaging can be performed as is customary in contemporary NMR experiments. This is demonstrated in Fig. $15(d)$, where a series of 16 spectra acquired over a period of $40 \mathrm{~min}$ from a sample of ${ }^{13} \mathrm{C}$ proline is shown. It illustrates that the instrumentation is sufficiently stable to reproduce the intensities in the spectra to $5 \%$.

\section{Pulsed DNP}

Apart from the pioneering work by Henstra et al. and Brunner et al., there have been few publications using the ISE or NOVEL reported. However, recently, it was shown that NOVEL can be used to enhance the ${ }^{13} \mathrm{C}$ NMR signal in diamonds. ${ }^{76}$ This was done at $9 \mathrm{GHz}$, corresponding to a ${ }^{13} \mathrm{C}$ Larmor frequency of $3.5 \mathrm{MHz}$. It was shown that the polarization rate of the ${ }^{13} \mathrm{C}$ nuclei in natural diamond is severely limited by the spin-diffusion bottleneck created by the slow rate at which flip-flop transitions occur among the diluted ${ }^{13} \mathrm{C}$ spins surrounding a paramagnetic impurity. Furthermore, the concentration of the paramagnetic impurities plays a dominant role in the polarization rate. A comparison of the ${ }^{13} \mathrm{C}$ polarization rates for the $\mathrm{SE}$ and NOVEL shows that, for the same microwave power, the polarization rates are comparable at higher impurity concentrations. However, at low impurity concentrations, the SE results in faster polarization of the ${ }^{13} \mathrm{C}$ nuclei.

\section{Future Perspectives}

Although high-field DNP has made significant progress towards evolving into a standard technique to study biological macromolecules, it remains a scientific field with a large potential for new developments both in theory and instrumentation.

Currently the water-soluble biradical TOTAPOL remains the only polarizing agent that can be used in high-field DNP to study biological systems. Although the enhancement factors are 
already very large, there is ample room for improvements, together with a deeper understanding of the DNP processes at high magnetic fields. For example, the synthesis of biradicals with more rigid tethers or radicals with narrower lines could produce even higher enhancements. Furthermore, a large number of biological macromolecules (e.g., electron transport proteins) have intrinsic paramagnetic centers, which have yet to be exploited as potential polarizing agents. In addition, a deeper understanding of the DNP processes at high magnetic fields for these species would be necessary.

The last two decades witnessed significant progress in microwave technology. Many new solidstate sources operating at high microwave frequencies are available with increasing output power and improvements in gyrotron technology will lead to more compact and practical designs.

Liquid DNP will benefit with new cavity designs, which minimize electric field heating and maximize $\mu \mathrm{w}$ penetration. These innovations will also have a large impact on high-field EPR and ENDOR spectroscopy.

Currently solid-state DNP experiments are performed at $90 \mathrm{~K}$ because of the experimental convenience of using liquid nitrogen as the cooling gas. However, lowering the temperature can further increase the efficiency of the polarization processes. MAS/NMR experiments at helium temperatures have been reported. ${ }^{123,124}$ and their incorporation into DNP experiments is in progress.

Finally, since classical cw DNP schemes suffer from an inverse field dependence, the implementation of DNP at higher magnetic fields should stimulate the development of new polarizing mechanisms potentially based on a coherent polarization transfer. In addition to these theoretical developments, time domain experiments will require $\mu \mathrm{w}$ amplifiers such as gyroamplifiers operating at frequencies of $140 \mathrm{GHz}$ and above to provide coherent high-power microwave pulses. With high $\mu \mathrm{w}$ power available, experiments such as the ISE, NOVEL, and DSSE will become possible or more efficient at high magnetic fields.

\section{Acknowledgments}

This research was supported by the National Institutes of Health through Grant Nos. EB002084, EB002026, EB009866, EB001965, and EB001035. T.M. acknowledges receipt of a postdoctoral fellowship of the Deutsche Forschungsgemeinschaft. The authors are grateful to Andrew B. Casey and Marvin J. Bayro for careful proofreading the manuscript. We thank Alexander Barnes for supplying Fig. 12 (right side).

\section{References}

1. Overhauser AW. Phys Rev 1953;92:411.

2. Carver TR, Slichter CP. Phys Rev 1953;92:212.

3. Carver TR, Slichter CP. Phys Rev 1956;102:975.

4. Abragam A, Proctor WG, Hebd CR. Seances Acad Sci 1958;246:2253.

5. Erb E, Motchane JL, Uebersfeld J, Hebd CR. Seances Acad Sci 1958;246:2121.

6. Kessenikh AV, Lushchikov VI, Manenkov AA, Taran YV. Sov Phys Solid State 1963;5:321.

7. Kessenikh AV, Manenkov AA, Pyatnitskii GI. Sov Phys Solid State 1964;6:641.

8. Hwang CF, Hill DA. Phys Rev Lett 1967;19:1011.

9. Hwang CF, Hill DA. Phys Rev Lett 1967;18:110.

10. Wollan DS. Phys Rev B 1976;13:3671.

11. Wind RA, Duijvestijn MJ, van der Luat C, Manenschijn A, Vriend J. Prog Nucl Magn Reson Spectrosc 1985;17:33.

12. Becerra L, Gerfen G, Temkin R, Singel D, Griffin R. Phys Rev Lett 1993;71:3561. [PubMed: 10055008] 
13. Bajaj V, Farrar C, Hornstein M, Mastovsky I, Vieregg J, Bryant J, Elena B, Kreischer K, Temkin R, Griffin R. J Magn Reson 2003;160:85. [PubMed: 12615147]

14. Hornstein MK, Bajaj VS, Griffin RG, Kreischer KE, Mastovsky I, Shapiro MA, Sirigiri JR, Temkin RJ. IEEE Trans Electron Devices 2005;52:798.

15. Farrar CT, Hall DA, Gerfen GJ, Inati SJ, Griffin RG. J Chem Phys 2001;114:4922.

16. Henstra A, Dirksen P, Schmidt J, Wenckebach WT. J Magn Reson 1988;77:389.

17. Henstra A, Dirksen P, Wenckebach WT. Phys Lett A 1988;134:134.

18. Henstra A, Lin TS, Schmidt J, Wenckebach WT. Chem Phys Lett 1990;165:6.

19. Weis V, Bennati M, Rosay M, Griffin RG. J Chem Phys 2000;113:6795.

20. Bennett LH, Torrey HC. Phys Rev 1957;108:499.

21. Hausser, KH.; Stehlik, D.; Waugh, JS., editors. Dynamic Nuclear Polarization in Liquids. Vol. 3. Academic; New York: 1968.

22. Mueller-Warmuth, W.; Meise-Gresch, K.; Waugh, JS., editors. Molecular Motions and Interactions as Studied by Dynamic Nuclear Polarization (DNP) in Free Radical Solutions. Vol. 11. Academic; New York: 1983.

23. Gitti R, Wild C, Tsiao C, Zimmer K, Glass TE, Dorn HC. J Am Chem Soc 1988;110:2294.

24. McCarney E, Armstrong B, Lingwood M, Han S. Proc Natl Acad Sci USA 2007; 104:1754. [PubMed: 17264210]

25. Ardenkjær-Larsen J, Fridlund B, Gram A, Hansson G, Hansson L, Lerche M, Servin R, Thaning M, Golman K. Proc Natl Acad Sci USA 2003;100:10158. [PubMed: 12930897]

26. Joo C, Hu K, Bryant J, Griffin R. J Am Chem Soc 2006;128:9428. [PubMed: 16848479]

27. Bowers CR, Weitekamp DP. J Am Chem Soc 1987;109:5541.

28. Bifone A, Song YQ, Seydoux R, Taylor RE, Goodson BM, Pietrass T, Budinger TF, Navon G, Pines A. Proc Natl Acad Sci USA 1996;93:12932. [PubMed: 8917521]

29. Schroder L, Lowery T, Hilty C, Wemmer D, Pines A. Science 2006;314:446. [PubMed: 17053143]

30. Blankenship R, McGuire A, Sauer K. Proc Natl Acad Sci USA 1975;72:4943. [PubMed: 174083]

31. Goldstein RA, Boxer SG. Biophys J 1987;51:937. [PubMed: 19431700]

32. Closs GL, Closs LE. J Am Chem Soc 1969;91:4549.

33. Zysmilich MG, McDermott A. J Am Chem Soc 1994;116:8362.

34. Deimling M, Brunner H, Dinse KP, Hausser KH, Colpa JP. J Magn Reson (1969-1992) 1980;39:185.

35. Schuhl A, Maegawa S, Meisel MW, Chapellier M. Phys Rev Lett 1985;54:1952. [PubMed: 10031184]

36. Ono K, Austing DG, Tokura Y, Tarucha S. Science 2002;297:1313. [PubMed: 12142438]

37. Ono K, Tarucha S. Phys Rev Lett 2004;92:256803. [PubMed: 15245046]

38. Sanada H, Matsuzaka S, Morita K, Hu CY, Ohno Y, Ohno H. Phys Rev Lett 2005;94:097601. [PubMed: 15783998]

39. Jeffries, CD. Dynamic Nuclear Orientation. Interscience; New York: 1963.

40. Abragam, A.; Goldman, M. Nuclear Magnetism: Order and Disorder. Clarendon, Oxford/Oxford University Press; New York: 1982.

41. Abragam A, Goldman M. Rep Prog Phys 1978;41:395.

42. Atsarkin VA. Sov Phys Usp 1978;21:725.

43. Poole, CP.; Farach, HA. Theory of Magnetic Resonance. Vol. 2nd. Wiley Interscience; New York: 1987.

44. Weil, JA.; Bolton, JR.; Wertz, JE. Electron Paramagnetic Resonance: Elementary Theory and Practical Applications. Wiley Interscience; New York: 1994.

45. Gordy, W. Theory and Applications of Electron Spin Resonance. Wiley; New York: 1980.

46. Freeman, AJ.; Frankel, RB. Hyperfine Interactions. Academic; New York: 1967.

47. Schweiger, A.; Jeschke, G. Principles of Pulse Electron Paramagnetic Resonance. Oxford University Press; Oxford, UK: 2001.

48. Kevan, L.; Bowman, MK. Modern Pulsed and Continuous-Wave Electron Spin Resonance. Wiley; New York: 1990.

49. Jeffries CD. Phys Rev 1957;106:164. 
50. Loening N, Rosay M, Weis V, Griffin R. J Am Chem Soc 2002;124:8808. [PubMed: 12137529]

51. Denninger G, Stocklein W, Dormann E, Schwoerer M. Mol Cryst Liq Cryst 1985;120:233.

52. Hoefer P, Parigi G, Luchinat C, Carl P, Guthausen G, Reese M, Carlomagno T, Griesinger C, Bennati M. Field dependent dynamic nuclear polarization (DNP) with radicals in aqueous solution. $\mathrm{J}$ Am Chem Soc. in press

53. Armstrong B, Han S. J Chem Phys 2007;127:104508. [PubMed: 17867762]

54. Grucker D, Guiberteau T, Eclancher B, Chambron J, Chiarelli R, Rassat A, Subra G, Gallez B. J Magn Reson 1995;106:101.B

55. Goldman, M. Spin Temperature and Nuclear Magnetic Resonance in Solids. Clarendon; Oxford: 1970.

56. Duijvestijn MJ, Wind RA, Smidt J. Physica B \& C 1986;138:147.

57. Wenckebach, WTh; Swanenburg, TJB.; Poulis, NJ. Phys Rev B 1974;14:181.

58. Hall D, Maus D, Gerfen G, Inati S, Becerra L, Dahlquist F, Griffin R. Science 1997;276:930. [PubMed: 9139651]

59. Hu K, Yu H, Swager T, Griffin R. J Am Chem Soc 2004;126:10844. [PubMed: 15339160]

60. Hu, Kan-Nian; Bajaj, Vikram S.; Rosay, Melanie; Griffin, Robert G. J Chem Phys 2007;126:044512. [PubMed: 17286492]

61. Song C, Hu K, Joo C, Swager T, Griffin R. J Am Chem Soc 2006;128:11385. [PubMed: 16939261]

62. Bajaj VS, Hornstein MK, Kreischer KE, Sirigiri JR, Woskov PP, Mak-Jurkauskas ML, Herzfeld J, Temkin RJ, Griffin RG. J Magn Reson 2007;190:86. [PubMed: 17981061]

63. van der Wel P, Hu K, Lewandowski J, Griffin R. J Am Chem Soc 2006;128:10840. [PubMed: 16910679]

64. Hartmann SR, Hahn EL. Phys Rev 1962;5:2042.

65. Pines A, Gibby MG, Waugh JS. J Chem Phys 1972;56:1776.

66. Conte P, Spaccini R, Piccolo A. Prog Nucl Magn Reson Spectrosc 2004;44:215.

67. Mehring, M. Principles of High-Resolution NMR in Solids. Vol. 2nd. Springer-Verlag; Berlin: 1983. 68. Bloembergen N, Sorokin PP. Phys Rev 1958;110:865.

69. Wind RA, Li L, Lock H, Maciel GE. J Magn Reson (1969-1992) 1988;79:577.

70. Wind RA, Lock H. Adv Magn Opt Reson 1990;15:51.

71. Farrar C, Hall D, Gerfen G, Rosay M, Ardenkjaer-Larsen J, Griffin R. J Magn Reson 2000;144:134. [PubMed: 10783283]

72. van den Heuvel DJ, Henstra A, Lin TS, Schmidt J, Wenckebach WT. Chem Phys Lett 1992;188:194.

73. Schmidt J, van den Heuvel DJ, Henstra A, Lin TS, Wenckebach WTh. Isr J Chem 1992;32:165.

74. Schmidt J, van den Heuvel DJ, Henstra A, Lin TS, Wenckebach WT. Pure Appl Chem 1992;64:859.

75. Brunner H, Fritsch RH, Hausser KH, Naturforsch Z. A: Phys Sci 1987;42:1456.

76. Reynhardt EC, High GL. J Chem Phys 1998;109:4100.

77. Takeda K, Takegoshi K, Terao T. Chem Phys Lett 2001;345:166.

78. Henstra, A.; Wenckebach, WT. Pulsed Magnetic Resonance: NMR, ESR and Optics. Bagguley, DMS., editor. Clarendon; Oxford: 1992. p. 411

79. Freed J. Annu Rev Phys Chem 2000;51:655. [PubMed: 11031296]

80. Weis V, Griffin R. Solid State Nucl Magn Reson 2006;29:66. [PubMed: 16298515]

81. Jeschke G, Schweiger A. J Chem Phys 1997;106:9979.

82. Diller A, Roy E, Gast P, van Gorkom H, de Groot H, Glaubitz C, Jeschke G, Matysik J, Alia A. Proc Natl Acad Sci USA 2007;104:12767. [PubMed: 17652174]

83. Prakash S, Alia A, Gast P, de Groot H, Jeschke G, Matysik J. Biochemistry 2007;46:8953. [PubMed: 17630781]

84. Dorn HC, Wang J, Allen L, Sweeney D, Glass TE. J Magn Reson (1969-1992) 1988;79:404.

85. Dorn HC, Gitti R, Tsai KH, Glass TE. Chem Phys Lett 1989;155:227.

86. Dorn HC, Gu J, Bethune DS, Johnson RD, Yannoni CS. Chem Phys Lett 1993;203:549.

87. Crabb DG, Meyer W. Annu Rev Nucl Part Sci 1997;47:67. 
88. Koelsch CF. J Am Chem Soc 1957;79:4439.

89. Ardenkjaer-Larsen J, Laursen I, Leunbach I, Ehnholm G, Wistrand L, Petersson J, Golman K. J Magn Reson 1998;133:1. [PubMed: 9654463]

90. Reddy T, Iwama T, Halpern H, Rawal V. J Org Chem 2002;67:4635. [PubMed: 12098269]

91. Afeworki M, McKay RA, Schaefer J. Macromolecules 1992;25:4084.

92. Anderson, S.; Golman, K.; Rise, F.; Wikstrom, H.; Wistrand, LG. U.S Patent No 5.530.140. 1996.

93. Granatstein VL, Parker RK, Armstrong CM. Proc IEEE 1999;87:702.

94. Abrams RH, Levush B, Mondelli AA, Parker RK. IEEE Microw Mag 2001;3:61.

95. Parker RK, Abrams RH Jr, Danly BG, Levush B. IEEE Trans Microwave Theory Tech 2002;50:835.

96. Hassan A, Pardi L, Krzystek J, Sienkiewicz A, Goy P, Rohrer M, Brunel L, Magn J. Reson 2000;142:300.

97. Barra AL. Appl Magn Reson 2001;21:619.

98. Prisner T, Un S, Griffin R. J Chem Phys 1992;32:357.

99. Grishin YA, Fuchs MR, Schnegg A, Dubinskii AA, Dumesh BS, Rusin FS, Bratman VL, Mobius K. Rev Sci Instrum 2004;75:2926.

100. Felch KL, Danly BG, Jory HR, Kreischer KE, Lawson W, Levush B, Temkin RJ. Proc IEEE 1999;87:752.

101. Nusinovich, GS. Introduction to the Physics of Gyrotrons. Johns Hopkins University Press; Baltimore: 2004.

102. Kartikeyan, MV.; Borie, E.; Thumm, MKA. Gyrotrons: High Power Microwave and Millimeter Wave Technology. Springer; Berlin: 2004.

103. Barker, RJ.; Schamiloglu, E. High-Power Microwave Sources and Technologies. IEEE; New York: 2001.

104. Cairns, RA.; Phelps, ADR. Generation and Application of High Power Microwaves: Proceedings of the Forty Eighth Scottish Universities Summer School in Physics, St Andrews, August 1996. Scottish Universities Summer School in Physics Institute of Physics Publishing; Edinburgh: 1997.

105. Gaponov-Grekhov, AV.; Granatstein, VL. Applications of High-Power Microwaves. Artech House; Boston: 1994.

106. Chu KR. Rev Mod Phys 2004;76:489.

107. Joye C, Griffin R, Hornstein M, Hu K, Kreischer K, Rosay M, Shapiro M, Siriqiri J, Temkin R, Woskov P. IEEE Trans Plasma Sci 2006;34:518.

108. Hornstein M, Bajaj V, Griffin R, Temkin R. IEEE Trans Plasma Sci 2006;34:524.

109. Becerra LR, Gerfen GJ, Bellew BF, Bryant JA, Hall DA, Inati SJ, Weber RT, Un S, Prisner TF, McDermott AE, Fishbein KW, Kreischer K, Temkin RJ, Singel DJ, Griffin RG. J Magn Reson 1995; 117:28.A

110. Doane, JL. Propagation and Mode Coupling in Corrugated and Smooth-Walled Circular Waveguides. Academic; New York: 1985.

111. Grinberg, OY.; Berliner, LJ.; Grinberg, OY.; Berliner, LJ., editors. Very High Frequency (VHF) ESR/EPR. Vol. 22. Kluwer Academic, Dordrecht/Plenum; New York: 2004.

112. Woskov PP, Bajaj VS, Hornstein MK, Temkin RJ, Griffin RG. IEEE Trans Microwave Theory Tech 2005;53:1863.

113. Grinberg OY, Dubinskii AA, Lebedev YS. Russ Chem Rev 1983;52:850.

114. Burghaus O, Rohrer M, Gotzinger T, Plato M, Mobius K. Meas Sci Technol 1992;3:765.

115. Weis V, Bennati M, Rosay M, Bryant J, Griffin R. J Magn Reson 1999;140:293. [PubMed: 10479576]

116. Hyde JS. J Chem Phys 1965;43:1806.

117. Gruber K, Forrer J, Schweiger A, Gunthard HH. J Phys E 1974;7:569.

118. Barnes A, De Paëpe G, van der Wel PCA, Hu K, Bajaj VS, Mak-Jurkauskas ML, Herzfeld J, Griffin RG. High field dynamic nuclear polarization for solid and solution biological NMR. Appl Magn Reson. submitted

119. Wind RA, Anthonio FE, Duijvestijn MJ, Smidt J, Trommel J, de Vette GMC. J Magn Reson (1969-1992) 1983;52:424. 
120. Cho H, Baugh J, Ryan C, Cory D, Ramanathan C. J Magn Reson 2007; 187:242. [PubMed: 17524687]

121. McKay, RA.; Grant, DM.; Harris, RK., editors. Probes for Special Purposes. Vol. 6. Wiley; Chichester: 1996.

122. Allen PJ, Creuzet F, de Groot HJM, Griffin RG. J Magn Reson (1969-1992) 1991;92:614.

123. Carravetta M, Johannessen OG, Levitt MH, Heinmaa I, Stern R, Samoson A, Horsewill AJ, Murata Y, Komatsu K. J Chem Phys 2006;124:104507. [PubMed: 16542088]

124. Macho V, Kendrick R, Yannoni CS. J Magn Reson (1969-1992) 1983;52:450.

125. Maly T, Bryant J, Ruben D, Griffin R. J Magn Reson 2006;183:303. [PubMed: 17027306]

126. Jacquinot JF, Wenckebach WT, Goldman M, Abragam A. Phys Rev Lett 1974;32:1096.

127. Afeworki M, Vega S, Schaefer J. Macromolecules 1992;25:4100.

128. Iijima T. Cryobiology 1998;36:165. [PubMed: 9597737]

129. Rosay M, Zeri A, Astrof N, Opella S, Herzfeld J, Griffin R. J Am Chem Soc 2001;123:1010. [PubMed: 11456650]

130. Rosay M, Lansing J, Haddad K, Bachovchin W, Herzfeld J, Temkin R, Griffin R. J Am Chem Soc 2003;125:13626. [PubMed: 14599177]

131. Rosay M, Weis V, Kreischer K, Temkin R, Griffin R. J Am Chem Soc 2002;124:3214. [PubMed: 11916398]

132. Sawaya M, Sambashivan S, Nelson R, Ivanova M, Sievers S, Apostol M, Thompson M, Balbirnie M, Wiltzius J, McFarlane H, Madsen A, Riekel C, Eisenberg D. Nature (London) 2007;447:453. [PubMed: 17468747]

133. Nelson R, Sawaya M, Balbirnie M, Madsen A, Riekel C, Grothe R, Eisenberg D. Nature (London) 2005;435:773. [PubMed: 15944695]

134. Maricq MM, Waugh JS. J Chem Phys 1979;70:3300.

135. Stevenson S, Dorn HC. Anal Chem 1994;66:2993.

136. Stevenson S, Glass T, Dorn HC. Anal Chem 1998;70:2623.

137. Russ JL, Gu J, Tsai KH, Glass T, Duchamp JC, Dorn HC. J Am Chem Soc. 2007

138. Granwehr J, Leggett J, Kockenberger W. J Magn Reson 2007;187:266. [PubMed: 17560151]

139. Frydman L, Scherf T, Lupulescu A. Proc Natl Acad Sci USA 2002;99:15858. [PubMed: 12461169]

140. Frydman L, Blazina D. Nat Phys 2007;3:415. 

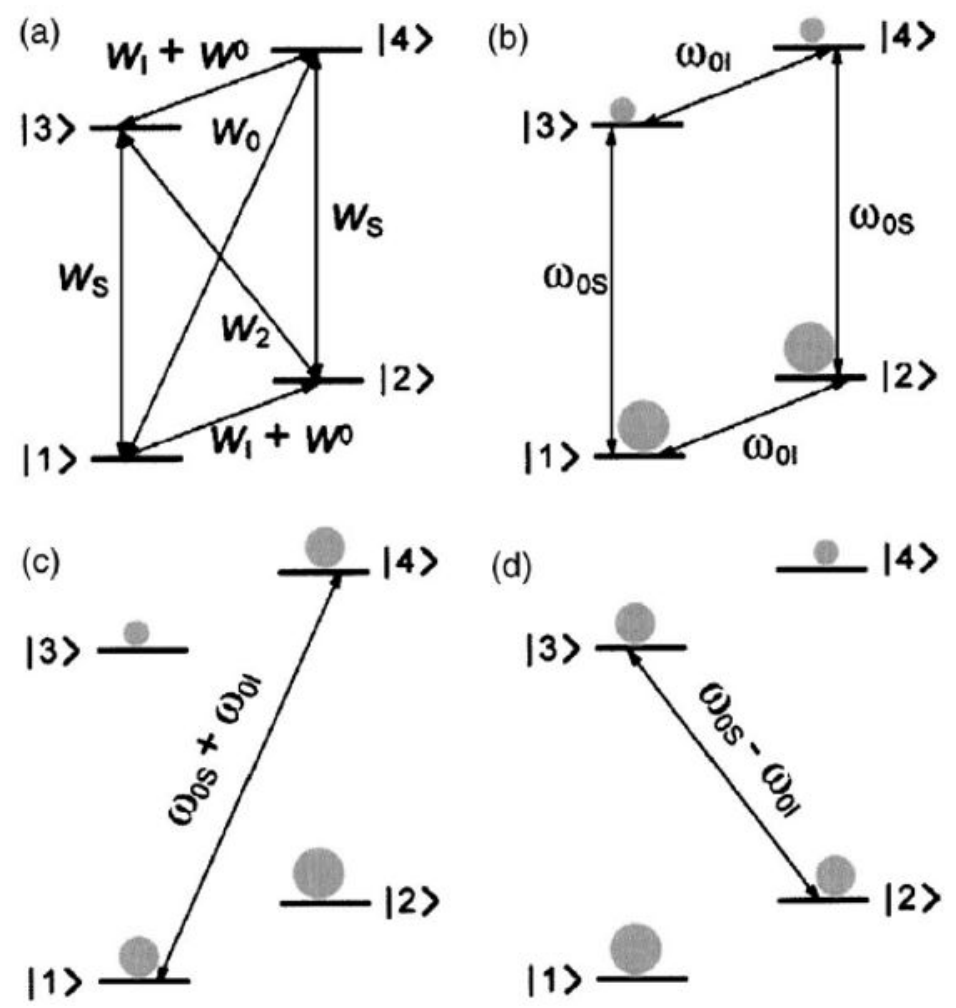

Fig. 1.

Energy level diagrams for the OE and SE. (a) Transition rates important for the OE. (b) Thermal equilibrium population for a two-level spin system. The spin population is depicted schematically in gray. [(c) and (d)] Saturation of the forbidden zero-quantum and doublequantum transitions leads to negative or positive enhancement through the SE. 


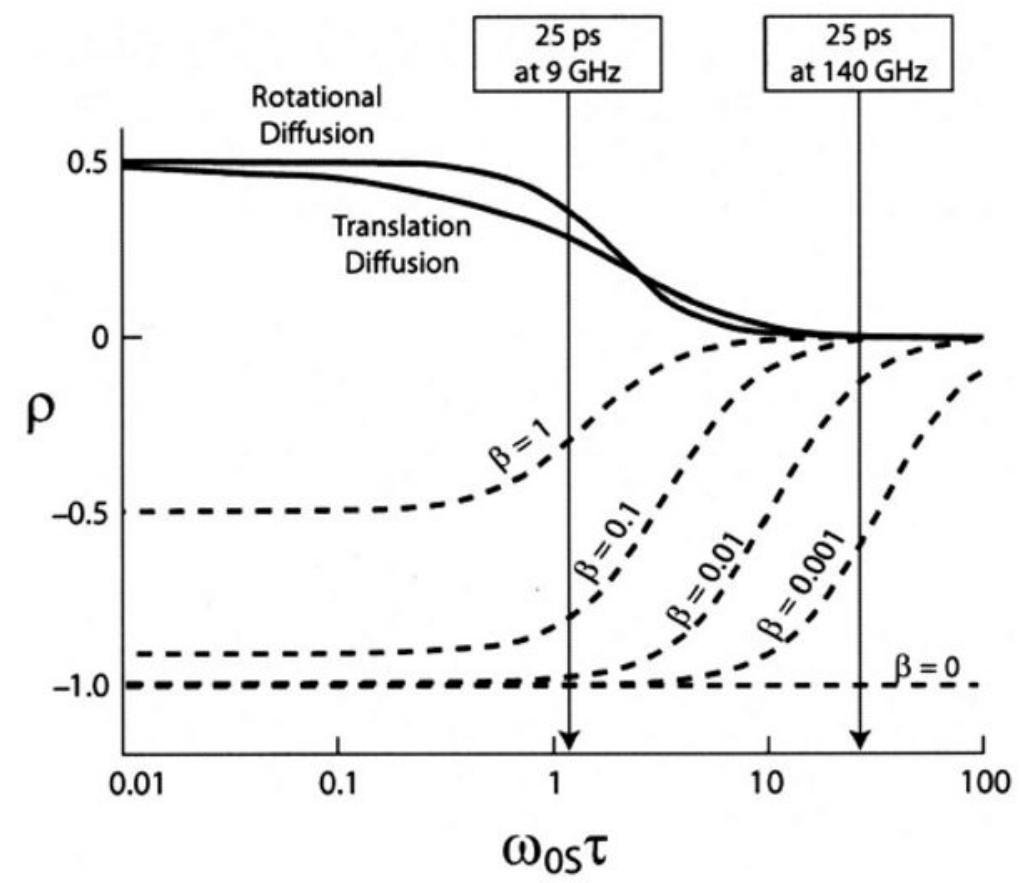

Fig. 2.

Dependence of the coupling parameter $\rho$ on the electron Larmor frequency $\omega_{0 S}$, the correlation time $\tau$, and the factor $\beta$. The arrows point out the difference in efficiency at 9 and $140 \mathrm{GHz}$ for $\tau=25$ ps. Figure adapted from Loening et al. (Ref. ${ }^{50}$ ). 


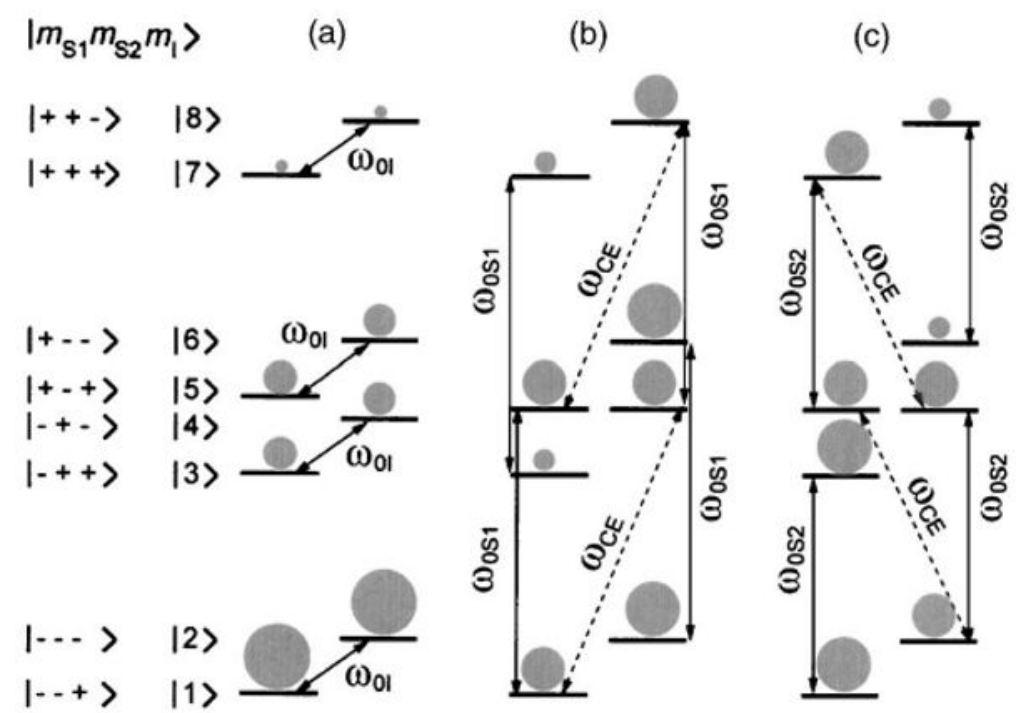

Fig. 3.

Population distribution at thermal equilibrium for a general three-spin system (a). Saturation of the allowed EPR transitions for one of the dipolar coupled electrons $\left(\omega_{0 S 1}\right)$ leads to negative enhancement (b). Saturation of the transition corresponding to the second electron $\left(\omega_{0 S 2}\right)$ leads to positive enhancement (c). 

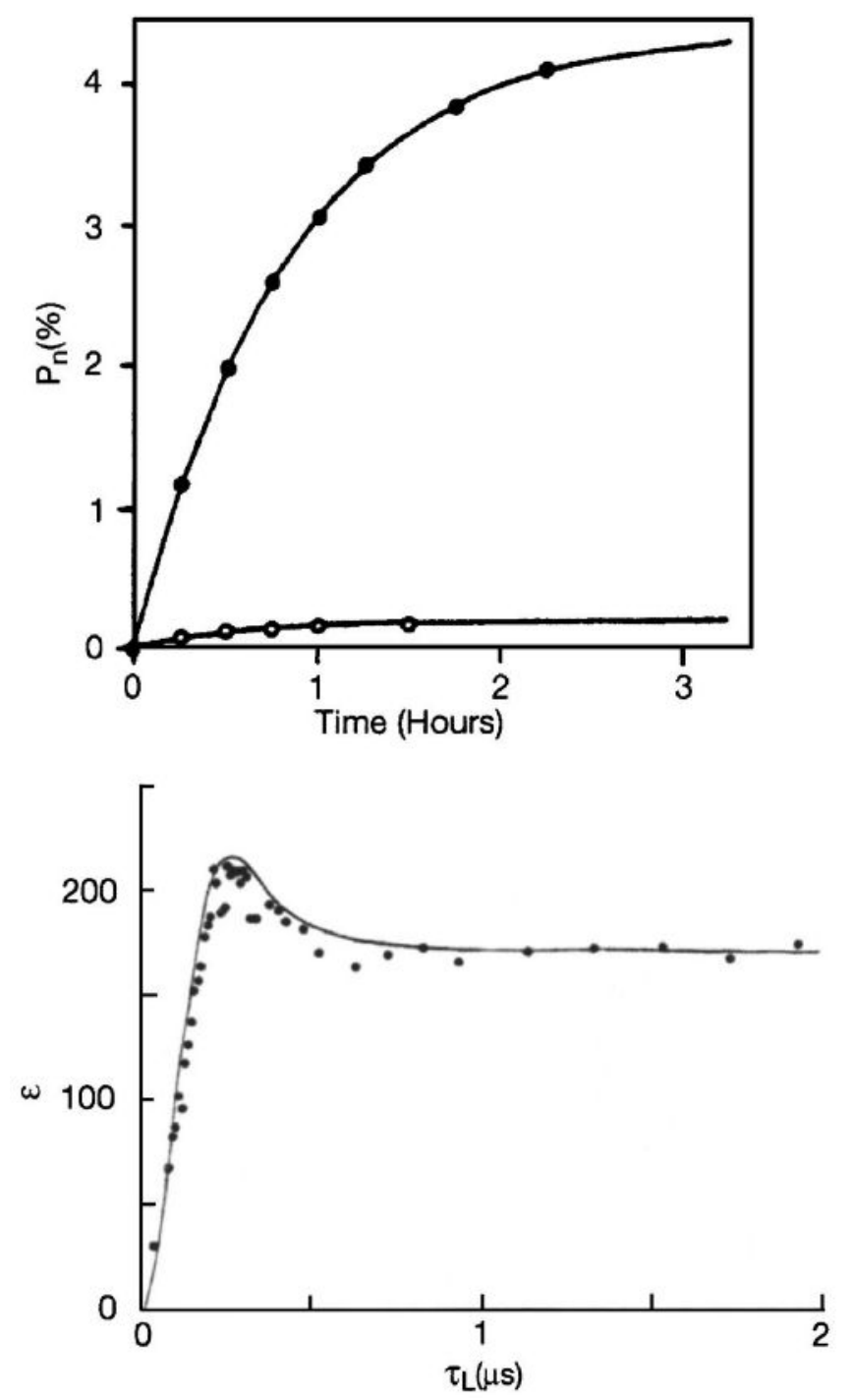

Fig. 4.

Top: Growth of the nuclear polarization (open circles-DSE, closed circles-ISE). Figure adapted from Henstra et al. (Ref. ${ }^{17}$ ). Bottom: Enhancement of the nuclear spin polarization as a function of the spin locking time $\tau_{L}$ in a NOVEL experiment. Figure adapted from Henstra et al. (Ref. 72 ). 


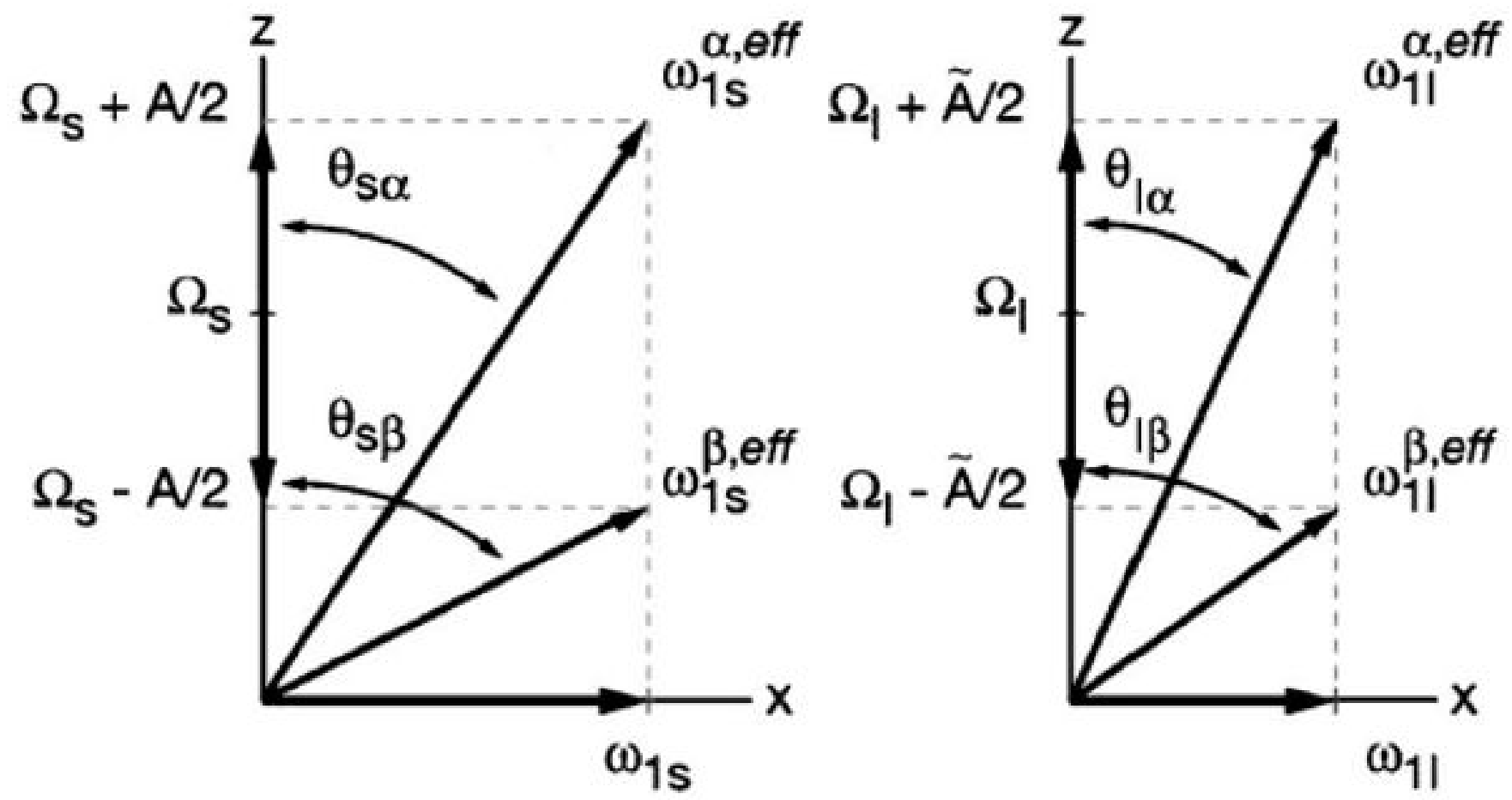

Fig. 5.

Illustration of the electron (S) and nuclear (I) spin effective fields. The effective fields belonging to the EPR and NMR transitions are no longer equal. Therefore, four effective fields are needed to accurately describe the two-spin system. 


\section{full mw power}
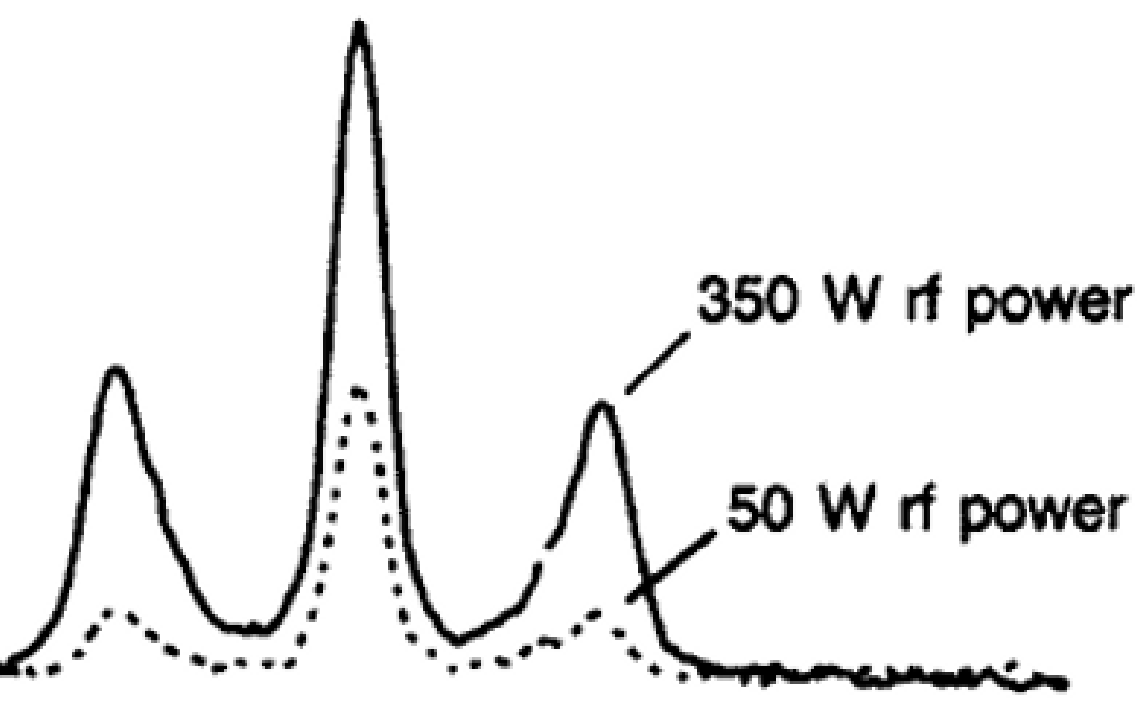

$-6 \mathrm{~dB}$ attenuation

$-12 \mathrm{~dB}$ attenuation

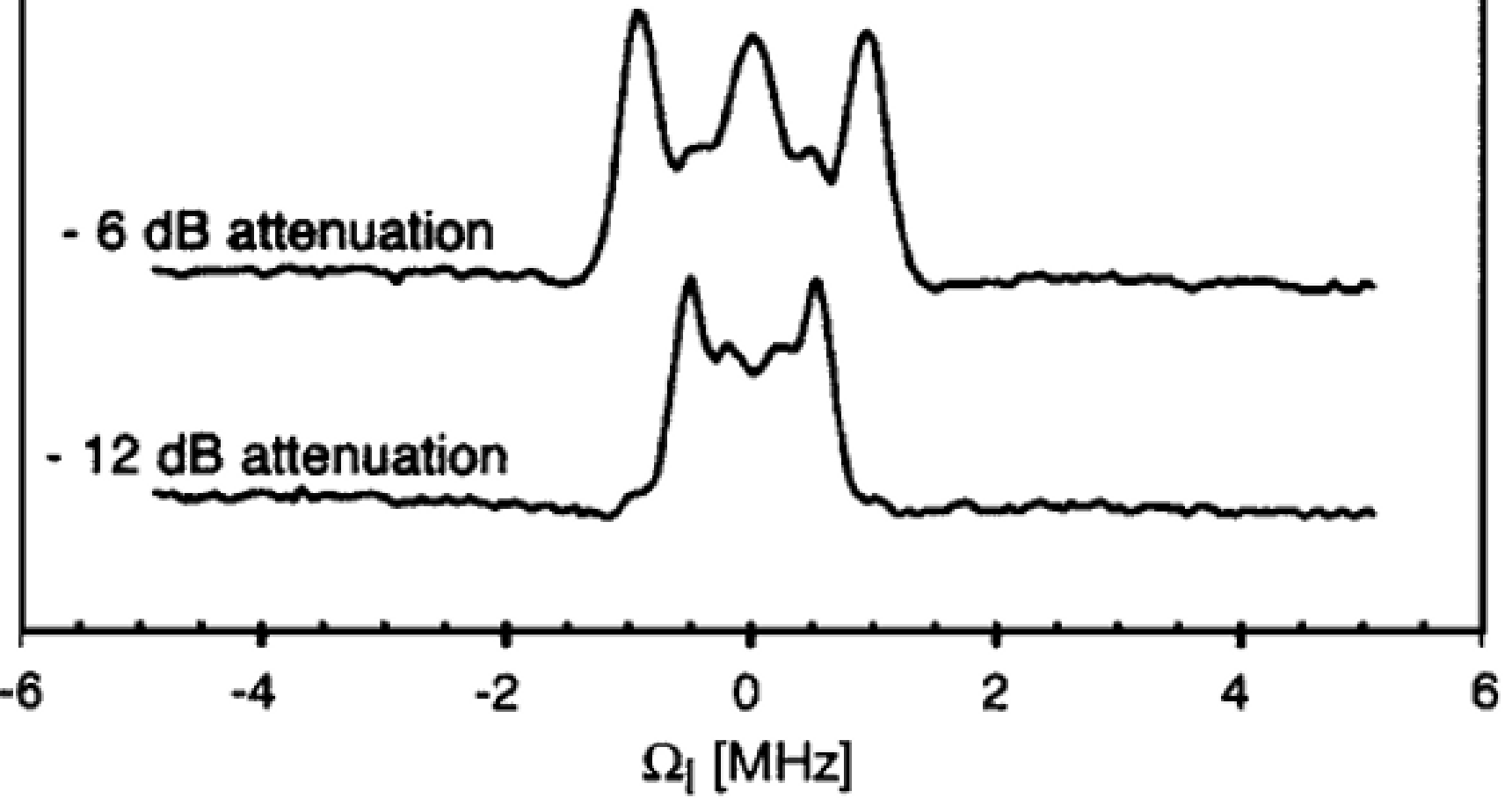

Fig. 6.

$140 \mathrm{GHz}$ eNCP experiment on perdeuterated BDPA for various settings of $\omega_{1 S}\left(\omega_{1 S} /\right.$ $2 \pi=1.75,0.9,0.5 \mathrm{MHz})$ and $\omega_{1 I}\left(\omega_{1 I} / 2 \pi=100 \mathrm{kHz}\right.$ at $\left.350 \mathrm{~W}\right)$. CP contact time was set to $3 \mu \mathrm{S}$. Figure adapted from Ref. 19 . 

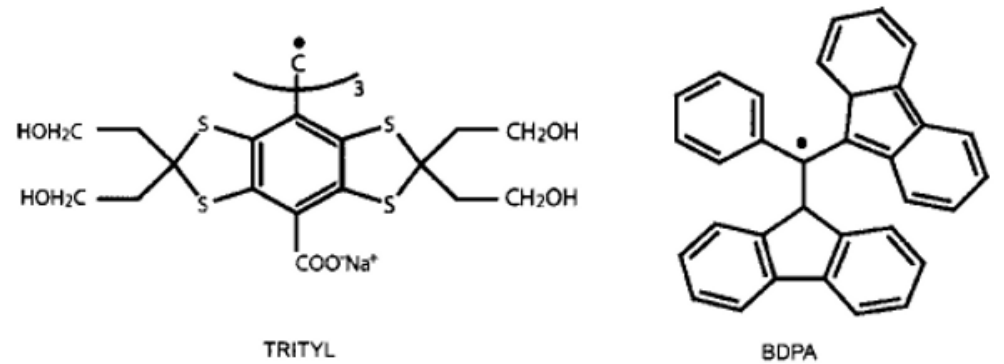

TRITYL

BDPA
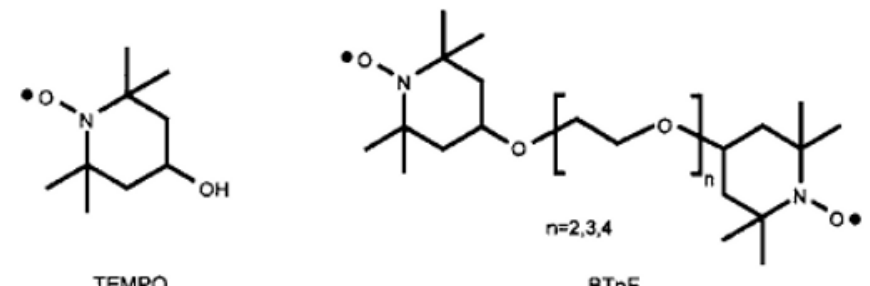

TEMPO

BTnE

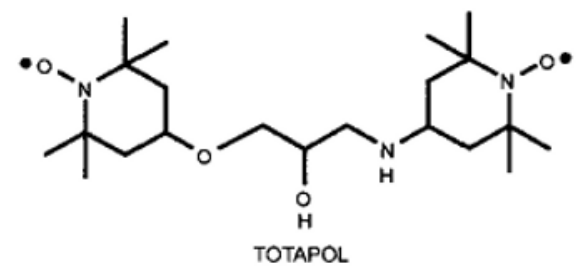

Fig. 7.

Chemical structures of three mono- and two biradical polarizing agents used in high-field DNP experiments. 

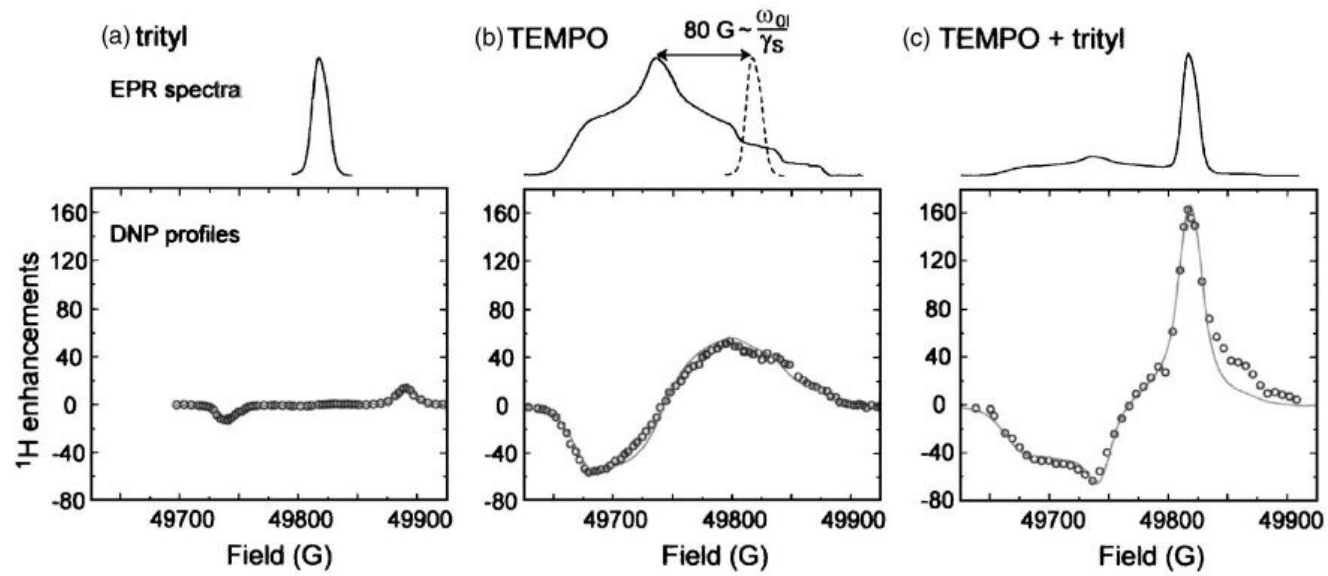

Fig. 8.

$140 \mathrm{GHz}$ EPR spectra and DNP field profiles for (a) trityl, (c) TEMPO, and (c) a mixture of trityl and TEMPO. The EPR spectra were recorded in ${ }^{2} \mathrm{H}_{6}-\mathrm{DMSO} /{ }^{2} \mathrm{H}_{2} \mathrm{O}$ 60:40 w/w $(1 \mathrm{mM})$ at $20 \mathrm{~K}$. The DNP samples were prepared in ${ }^{2} \mathrm{H}_{6}-\mathrm{DMSO} /{ }^{2} \mathrm{H}_{2} \mathrm{O} / \mathrm{H}_{2} \mathrm{O}$ 60:34:6 w/w/w with a total radical concentration of $40 \mathrm{mM}(90 \mathrm{~K})$. The solid line represents a simulation of the experimental data. Figure taken from $\mathrm{Hu}$ et al. $\left({ }^{\text {Ref. } 60}\right)$. 

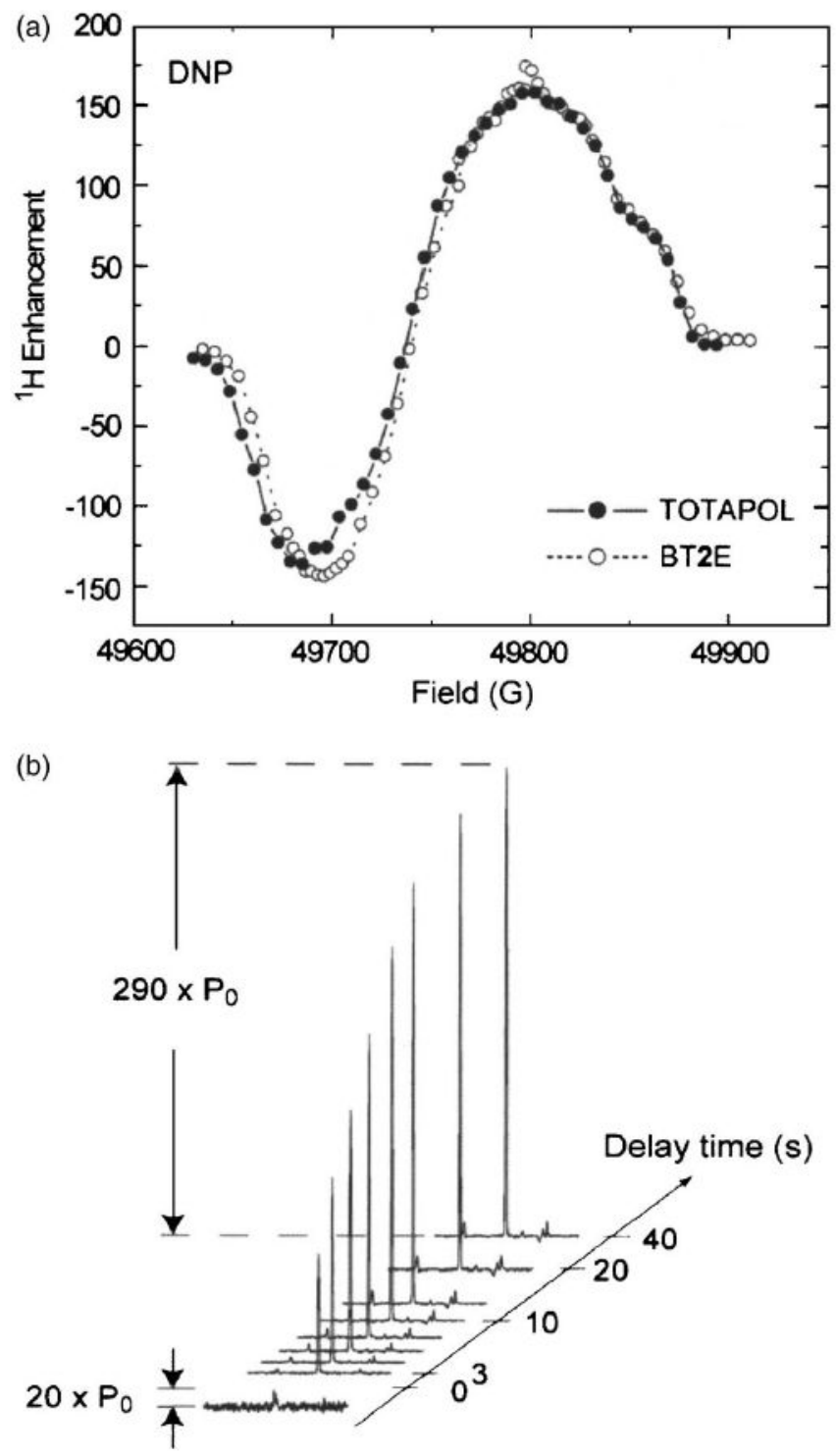

Fig. 9.

(a) Field profile of TOTAPOL and BT2E recorded at $5 \mathrm{~T}$ (90 K, $4 \mathrm{~mm}$ rotor). (b) Enhancement profile of ${ }^{13} \mathrm{C}$-urea $(2 M)$ as a function of the $\mathrm{MW}$ irradiation time in the presence of $3 \mathrm{mM}$ TOTAPOL (2.5 mm rotor). Figures taken from Song et al. (Ref. 61). 


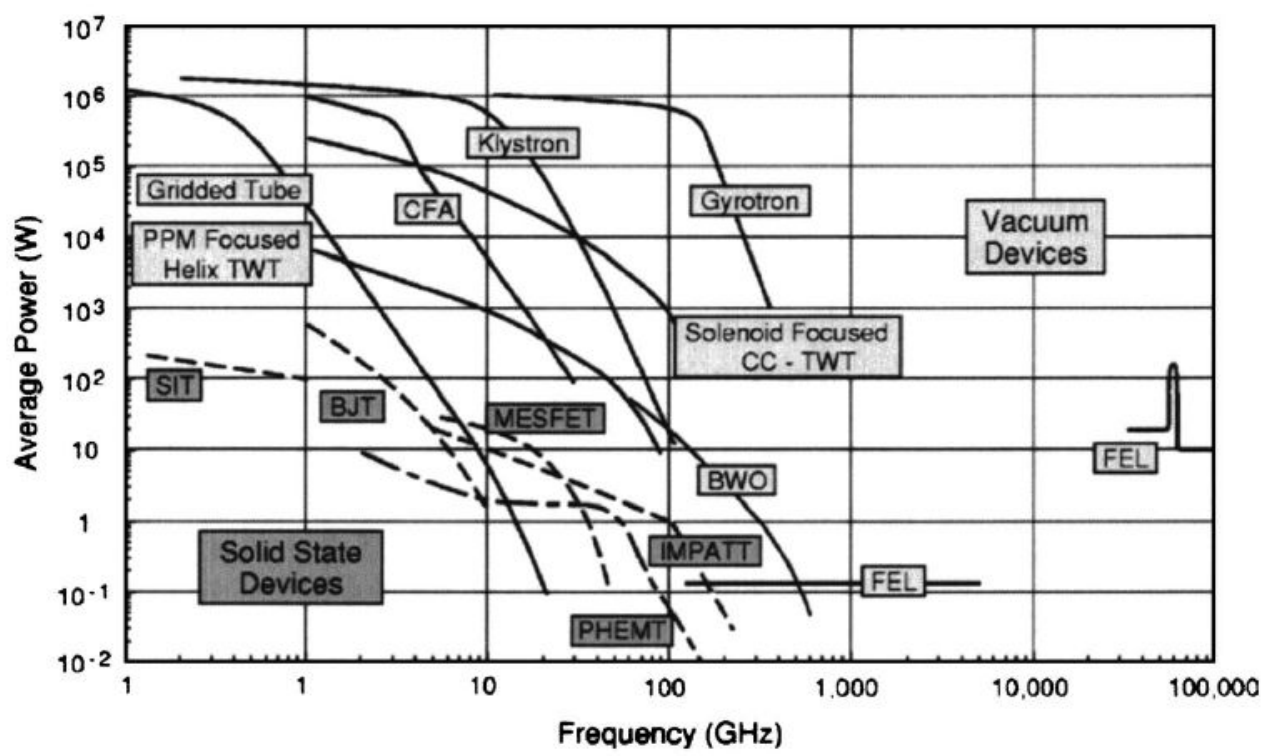

Fig. 10.

Present state of vacuum electronic devices in terms of the ability of multiple devices types to generate average power at a certain frequency. Figure taken from Granatstein et al. $\left({ }^{\text {Ref. }}{ }^{93}\right)$. 


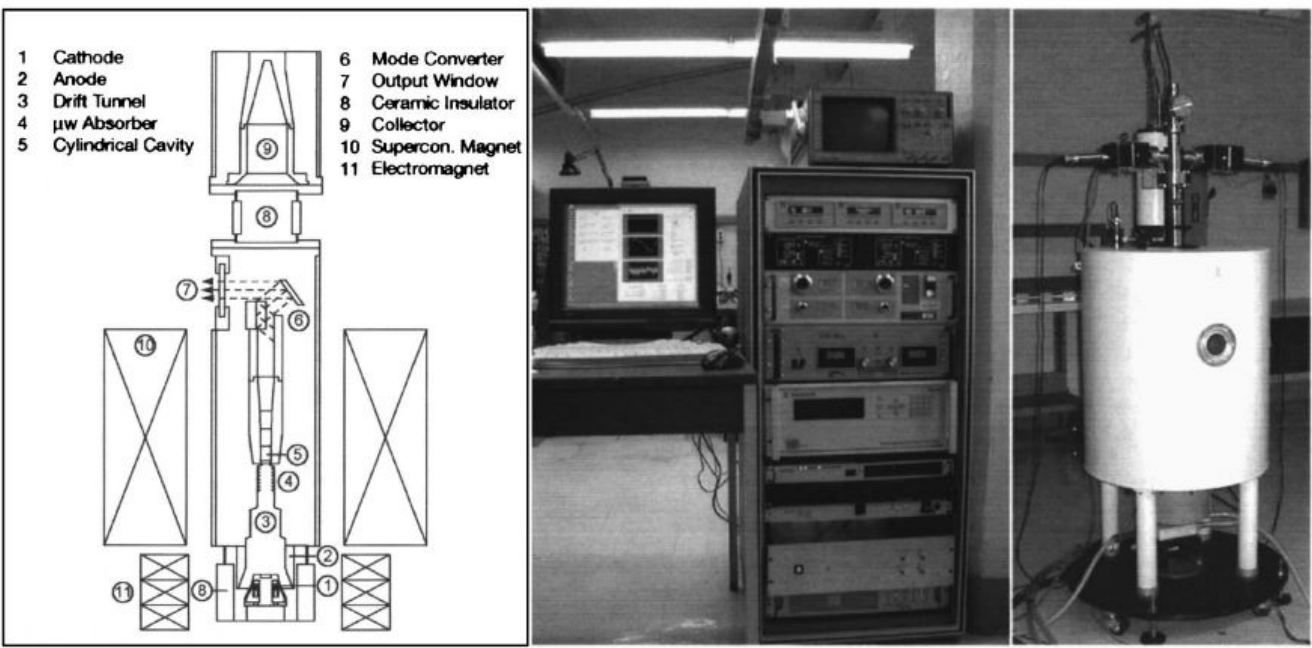

Fig. 11.

Left: Schematic of a gyrotron tube indicating its key components. Figure taken from Hornstein et al. $\left({ }^{\text {Ref. }}{ }^{14}\right)$. Middle and right: Photographs of the $460 \mathrm{GHz}$ gyrotron. 

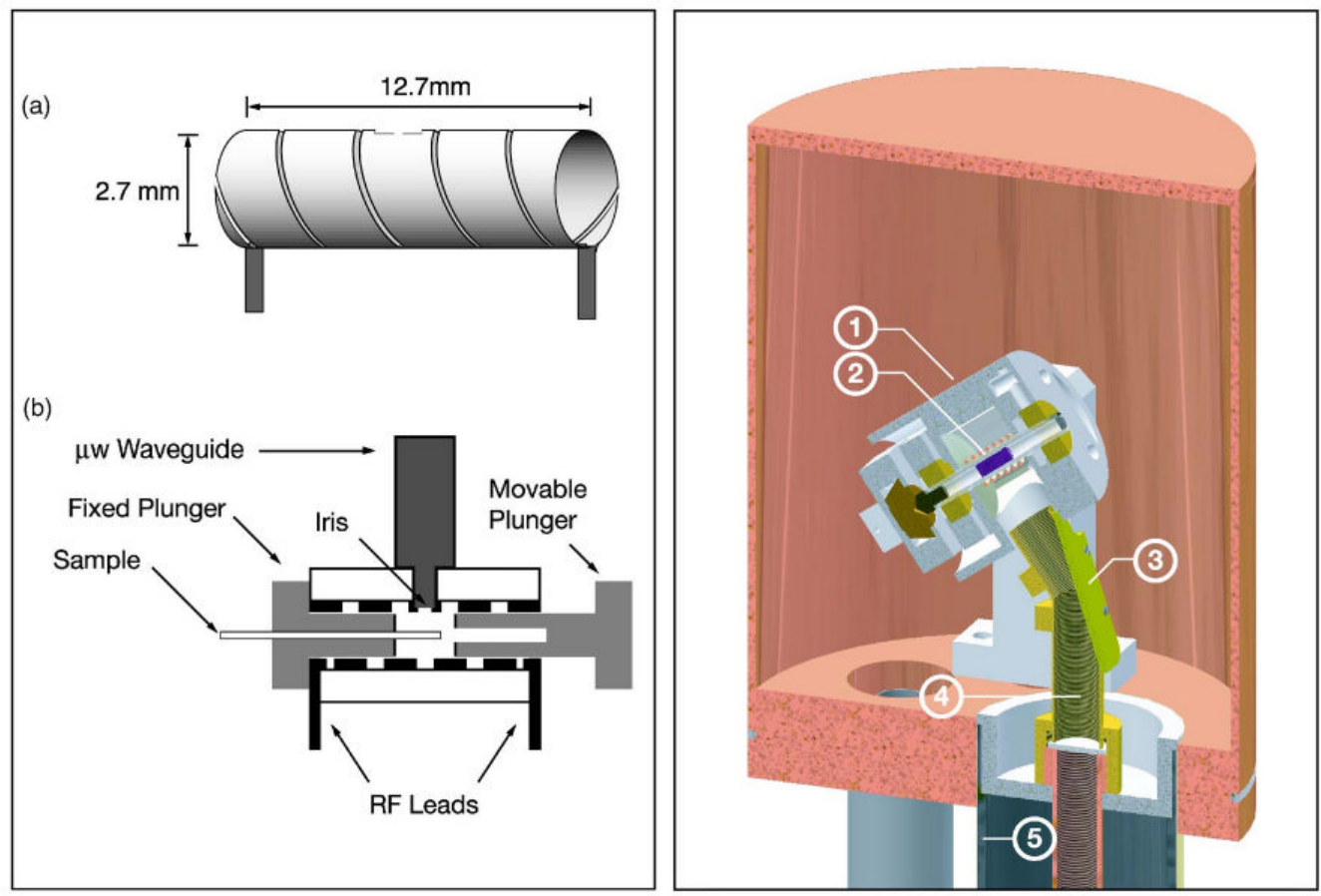

Fig. 12.

(Color online) Layout of DNP probes. Left: Probe used for DNP in liquids and solids. Figure adapted from Weis et al. (Ref. 115). Right: Low temperature MAS probe for ssNMR DNP experiments. (1) Stator, (2) Sample, (3) Miter bend, (4) Inner conductor, and (5) Outer conductor. Figure adapted from Barnes et al. $\left({ }^{\text {Ref. }}{ }^{118}\right)$. 


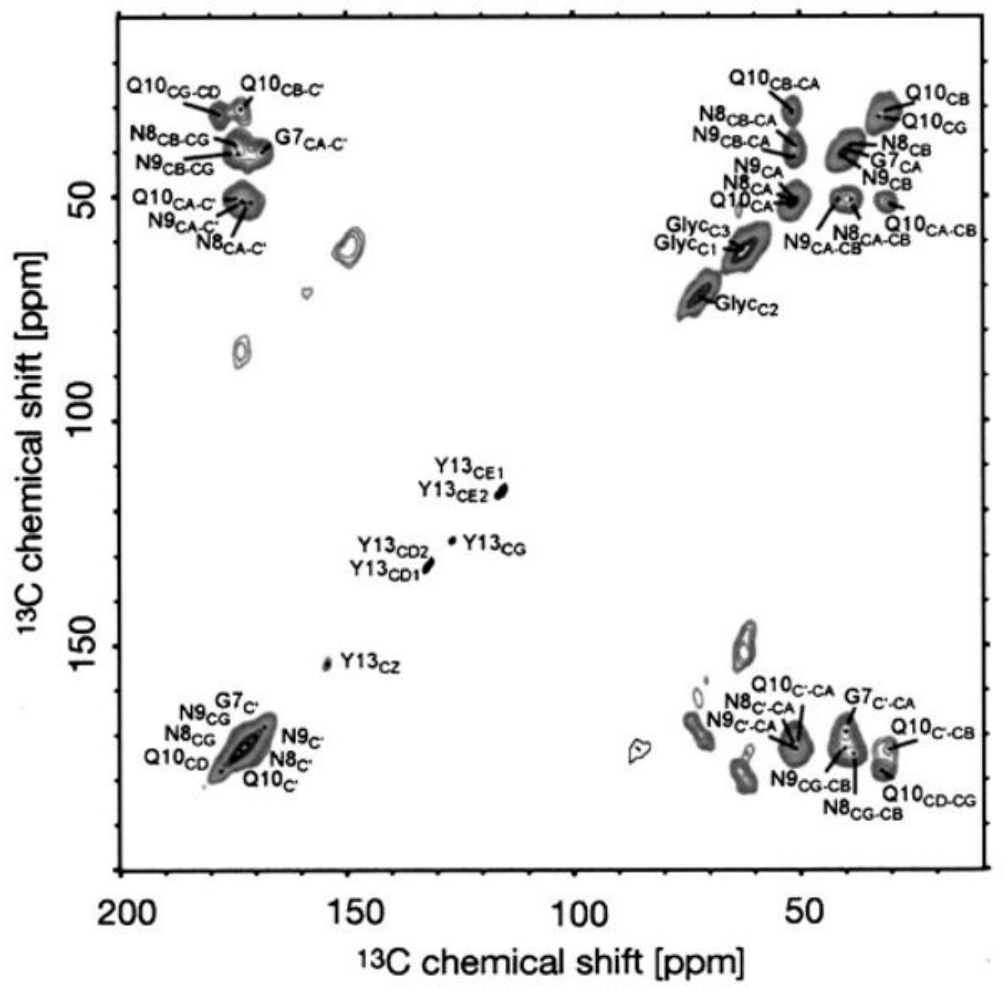

Fig. 13.

DNP-enhanced ${ }^{13} \mathrm{C}-{ }^{13} \mathrm{C}$ DARR/RAD correlation spectrum of [20\% $\mathrm{U}-{ }^{13} \mathrm{C},{ }^{15} \mathrm{~N}-\mathrm{GNNQ}$ ] QNY nanocrystals. Figure adapted from van der Wel et al. $\left({ }^{\text {Ref. } 63}\right)$. 


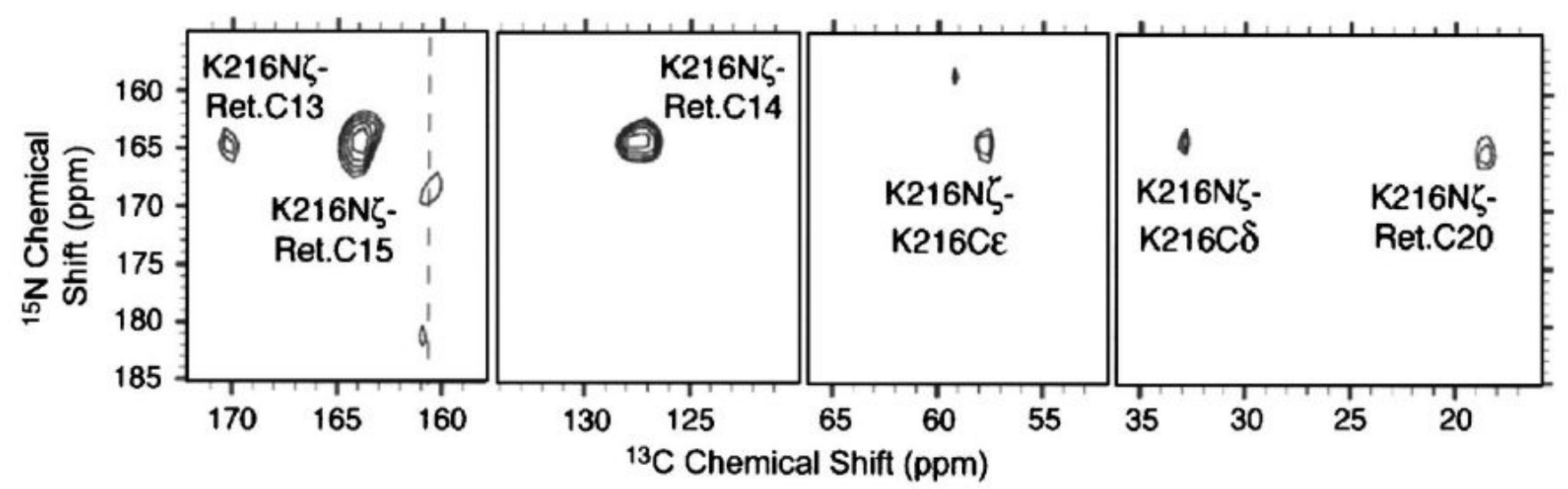

Fig. 14.

Schiff base region of a typical 2D Lys-N $\zeta$-Ret.-C15-CX correlation spectrum (DAR/RAAD) of $\left[\mathrm{U}-{ }^{13} \mathrm{C},{ }^{15} \mathrm{~N}\right]-\mathrm{bR}$ in the light adapted state $\left(\mathrm{bR}_{568}\right)$. Multiple chemical shift assignments result from a single experiment. $\omega_{r} / 2 \pi=7 \mathrm{kHz}$. Figure taken from Bajaj et al. (Ref. 62 ). 
(a)

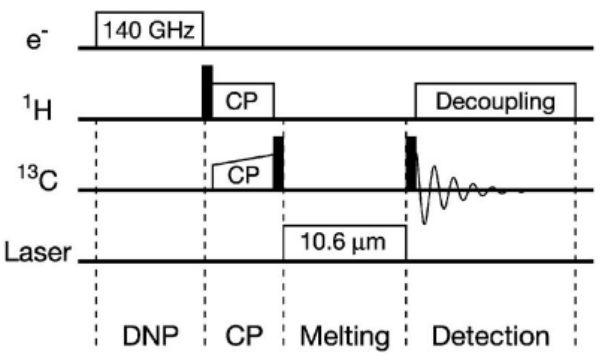

(c)

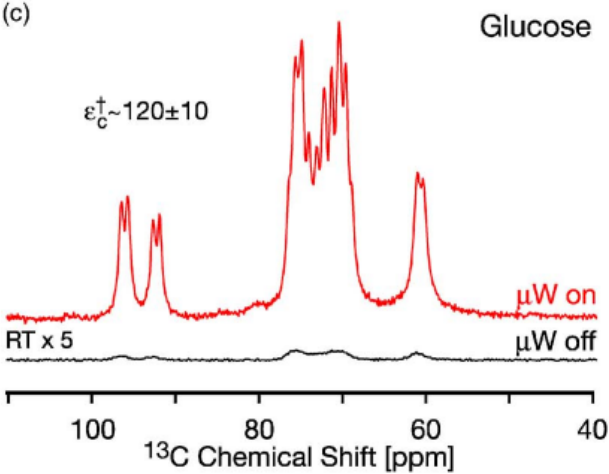

(b)

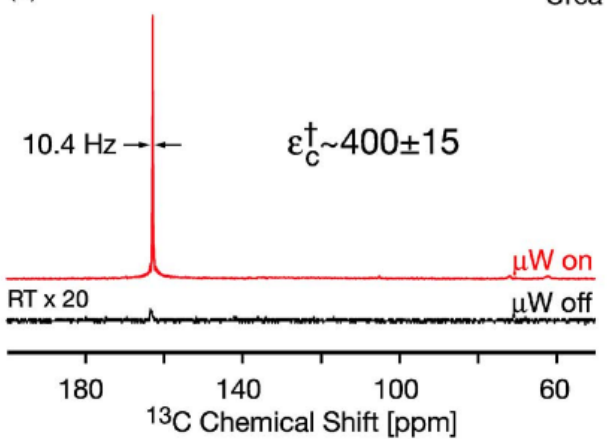

(d)

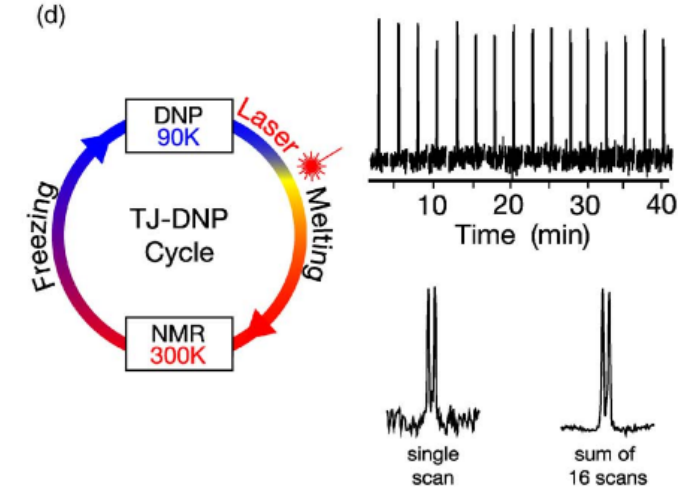

Fig. 15.

(Color online) In situ TJ-DNP experiment. (a) Pulse sequence. (b) ${ }^{13} \mathrm{C}-\mathrm{TJ}-\mathrm{DNP}$ NMR spectra of ${ }^{13} \mathrm{C}$-urea in $50 \%{ }^{2} \mathrm{H}_{6}$-DMSO and $50 \%$ water $\left({ }^{2} \mathrm{H}_{2} \mathrm{O} / \mathrm{H}_{2} \mathrm{O}=4 / 1\right)$. (c) ${ }^{13} \mathrm{C}$-TJ-DNP NMR spectra of $\left[\mathrm{U}-{ }^{13} \mathrm{C}_{6},{ }^{2} \mathrm{H}_{7}\right]$-glucose in $\mathrm{H}_{2} \mathrm{O}$. D: 16 spectra of the $\mathrm{CO}$ resonance in $\left[\mathrm{U}-{ }^{13} \mathrm{C}\right]-$ $L$-proline resulting from a series of TJ-DNP experiments. (Below) average of the 16 spectra giving an improved signal-to-noise ratio. Samples contained 3-5 mM TOTAPOL biradical polarizing agent corresponding to $6-10 \mathrm{mM}$ electrons. The TJ-DNP spectra (the top traces in each figure) were recorded with a single scan, while the RT spectra were recorded with 256 (a) and 512 scans (b). 